\title{
SHORT MATURITY ASIAN OPTIONS IN LOCAL VOLATILITY MODELS
}

\author{
DAN PIRJOL AND LINGJIONG ZHU
}

\begin{abstract}
We present a rigorous study of the short maturity asymptotics for Asian options with continuous-time averaging, under the assumption that the underlying asset follows a local volatility model. The asymptotics for out-of-the-money, in-the-money, and at-the-money cases are derived, considering both fixed strike and floating strike Asian options. The asymptotics for the out-of-the-money case involves a non-trivial variational problem which is solved completely. We present an analytical approximation for Asian options prices, and demonstrate good numerical agreement of the asymptotic results with the results of Monte Carlo simulations and benchmark test cases in the Black-Scholes model for option parameters relevant in practical applications.
\end{abstract}

\section{INTRODUCTION}

Asymptotics of the option prices, and implied volatility for short maturity European options have been extensively studied in the literature, see e.g. [4, 29, 30, 19, 8, for the local volatility models, 22, 46, 3, 18, 43 for the exponential Lévy models and [5, 36, 25, 20, 21, 23, 1] for the stochastic volatility models and [28, 40] for model-free approaches. To the best of our knowledge, the short maturity Asian options have been much less studied. Unlike the European options, the Asian options do not have a simple closed-form formula even in the Black-Scholes model. That is why in financial industry, the Asian options are quoted by price rather than implied volatility. In this paper, we study the short maturity asymptotics for the price of the Asian options under the assumption that the underlying asset price follows a local volatility model. We obtain analytical results for the short maturity asymptotics of Asian options in the local volatility model, and more explicit results in the case of the Black-Scholes model. We define and study the implied volatility of an Asian option in the short maturity limit.

Let us assume that the stock price follows a local volatility model:

$$
d S_{t}=(r-q) S_{t} d t+\sigma\left(S_{t}\right) S_{t} d W_{t}, \quad S_{0}>0,
$$

where $W_{t}$ is a standard Brownian motion, $r \geq 0$ is the risk-free rate, $q \geq 0$ is the continuous dividend yield, $\sigma(\cdot)$ is the local volatility and the log-stock price process $X_{t}=\log S_{t}$ satisfies

$$
d X_{t}=\left(r-q-\frac{1}{2} \sigma^{2}\left(e^{X_{t}}\right)\right) d t+\sigma\left(e^{X_{t}}\right) d W_{t}
$$

Date: 19 September 2016.

2010 Mathematics Subject Classification. 91G20,91G80,60F10.

Key words and phrases. Asian options, short maturity, local volatility, large deviations, variational problem. 
We assume that the local volatility function $\sigma(\cdot)$ satisfies

$$
\begin{aligned}
& 0<\underline{\sigma} \leq \sigma(\cdot) \leq \bar{\sigma}<\infty, \\
& \left|\sigma\left(e^{x}\right)-\sigma\left(e^{y}\right)\right| \leq M|x-y|^{\alpha},
\end{aligned}
$$

for some fixed $M, \alpha>0$ for any $x, y$ and $0<\underline{\sigma}<\bar{\sigma}<\infty$ are some fixed constants.

The price of the Asian call and put options with maturity $T$ and strike $K$ are given by

$$
\begin{aligned}
& C(T):=e^{-r T} \mathbb{E}\left[\left(\frac{1}{T} \int_{0}^{T} S_{t} d t-K\right)^{+}\right], \\
& P(T):=e^{-r T} \mathbb{E}\left[\left(K-\frac{1}{T} \int_{0}^{T} S_{t} d t\right)^{+}\right],
\end{aligned}
$$

where $C(T)$ and $P(T)$ emphasize the dependence on the maturity $T$.

When $S_{0}<K$, the call option is out-of-the money and $C(T) \rightarrow 0$ as $T \rightarrow 0$ and when $S_{0}>K$, the put option is out-of-the money and $P(T) \rightarrow 0$ as $T \rightarrow 0$. When $S_{0}=K$, i.e. at-the-money, both $C(T)$ and $P(T)$ tend to zero as $T \rightarrow 0$. We are interested to study the first-order approximations of the call and put prices as $T \rightarrow 0$. It turns out that the asymptotics for out-of-the-money case are governed by the rare events (large deviations) and the asymptotics for at-the-money case are governed by the fluctuations about the typical events (Gaussian fluctuations).

There are numerous works in the mathematical finance literature studying the pricing of Asian options. The pricing under the Black-Scholes model has been studied in [31, 7, 14, 39], using a relation between the distributional property of the time-integral of the geometric Brownian motion and Bessel processes. See [16] for an overview, and [27] for a comparison with alternative simulation methods, including the Monte Carlo approach. A popular method, which has the advantage of wider applicability to other models, is the PDE approach [37, 42, 49, 50]. The resulting PDE can be solved either numerically [49, 50, or can be used to derive analytical approximation formulae using asymptotic expansion methods. Such results have been obtained in [26] in the local volatility model, and in [1] in the CEV model. The paper [26] used heat kernel expansion methods and developed approximate formulae expressed in terms of elementary functions for the density, the price and the Greeks of path dependent options of Asian style. Asymptotic expansion leading to analytical approximations with error bounds for Asian options have been obtained also using Malliavin calculus in [45, 32].

Most of the literature on Asian options in the Black-Scholes model, i.e., $\sigma(\cdot) \equiv \sigma, 31$, 7, 39] exploits the well-known result [16, 13] that the integral of the geometric Brownian motion $\int_{0}^{T} e^{\left(r-q-\frac{1}{2} \sigma^{2}\right) t+\sigma W_{t}} d t$ has the same distribution as $X_{t}$, where

$$
d X_{t}=\left[(r-q)+\frac{1}{X_{t}}\right] X_{t} d t+\sigma X_{t} d B_{t}, \quad X_{0}=0
$$

where $B_{t}$ is a standard Brownian motion. The price of the Asian call and put options can thus be computed as

$$
C(T)=e^{-r T} \frac{1}{T} \mathbb{E}\left[\left(S_{0} X_{T}-T K\right)^{+}\right], \quad P(T)=e^{-r T} \frac{1}{T} \mathbb{E}\left[\left(T K-S_{0} X_{T}\right)^{+}\right] .
$$

For the readers who are familiar with the large deviations for small time diffusion processes, one may naïvely believe that the small time asymptotics of $X_{T}$ as $T \rightarrow 0$ is comparable 
with the SDE without the drift term, i.e., $d \tilde{X}_{t}=\sigma \tilde{X}_{t} d \tilde{B}_{t}$ and hence the asymptotics for the short maturity Asian options for the Black-Scholes model are the same as for their European counterpart. We will show in this paper that this is indeed not the case. Intuitively, when $\tilde{X}_{t}$ starts at zero at time zero then $\tilde{X}_{t}$ remains zero for any time $t$. Even though $X_{t}$ also starts at zero at time zero, it is kicked to a positive value immediately after the time zero. In that respect, the two processes are not absolutely continuous with respect to each other. Therefore, one cannot use the Girsanov theorem to kill the drift term and claim that $X_{t}$ process has the same small time asymptotics as a geometric Brownian motion. Also, the formulas (6), (7) are valid only for the Black-Scholes model, and this approach does not shed much insight into the more general local volatility case.

In this paper, we will use large deviations theory for small time diffusion processes. The key observation is that one can apply the contraction principle to get the corresponding large deviations for the small time arithmetic average of the diffusion process, and hence obtain rigorously the asymptotic behavior for the out-of-the-money Asian call and put options. The asymptotic exponent is given as the rate function from the large deviation principle, which itself is a complicated and not-so-obvious variational problem. We will manage to solve this variational problem completely and give a semi-analytical solution in the end. The asymptotics for in-the-money case follows easily by the put-call parity. We will also obtain the asymptotics for at-the-money short maturity Asian options. Unlike the out-of-the-money case, the asymptotics for at-the-money short maturity has Gaussian fluctuations.

Most of the existing methods for pricing Asian options are numerically less efficient in the limit of small maturities and small volatilities. For the case of the Asian options under the Black-Scholes model this has been noted in the Geman-Yor method [31, 7], where the inversion of a Laplace transform requires special care for small maturities [44, 27, 14]. A similar issue appears in the spectral method [39]. This issue is not present in methods based on asymptotic expansions [26] which perform well under small maturities and volatility conditions. The small time expansion presented in this paper is of practical interest as it complements some of the alternative approaches in a region where their numerical performance is less efficient.

The paper is organized as follows. In Section 2, we present asymptotics for out-of-themoney (OTM), in-the-money (ITM) and at-the-money (ATM) Asian options in a local volatility model for short maturities. The asymptotics for OTM Asian options involve a not-so-trivial variational problem, whose solution will be given in Section 3, which has a more explicit expression in the case of Black-Scholes models. The implied volatility and numerical tests will be discussed in Section 4. The asymptotics for short maturity floating strike Asian options will be provided in Section 5. Finally, the proofs will be given in Section 6 .

\section{Asymptotics for Short Maturity Asian Options}

Let us recall that the stock price follows a local volatility model:

$$
d S_{t}=(r-q) S_{t} d t+\sigma\left(S_{t}\right) S_{t} d W_{t}, \quad S_{0}>0,
$$

where $W_{t}$ is a standard Brownian motion, $r \geq 0$ is the risk-free rate, $q \geq 0$ is the continuous dividend yield, $\sigma(\cdot)$ is the local volatility satisfying (2) and (3). We are interested in the short maturity limits, i.e., the asymptotics as $T \rightarrow 0$. 
2.1. Out-of-the-Money and In-the-Money Asian Options. We denote the expectation of the averaged asset price in the risk-neutral measure as

$$
A(T):=\frac{1}{T} \int_{0}^{T} \mathbb{E}\left[S_{t}\right] d t=S_{0} \frac{1}{(r-q) T}\left(e^{(r-q) T}-1\right),
$$

for $r-q \neq 0$ and $A(T):=S_{0}$ for $r-q=0$, When $K>A(T)$, the call Asian option is out-of-the-money and $C(T) \rightarrow 0$ as $T \rightarrow 0$. When $A(T)>K$, the put Asian option is out-of-the-money and $P(T) \rightarrow 0$ as $T \rightarrow 0$.

Remark 1. The prices of call and put Asian options are related by put-call parity as

$$
C(K, T)-P(K, T)=e^{-r T}(A(T)-K) .
$$

Notice that as $T \rightarrow 0, A(T)=S_{0}+O(T)$. Therefore, for the small maturity regime, the call Asian option is out-of-the-money if and only if $K>S_{0}$ etc. And for the rest of the paper, the call Asian option is said to be out-of-the-money (resp. in-the-money) if $K>S_{0}$ (resp. $K<S_{0}$ ), and the put Asian option is said to be out-of-the-money (resp. in-the-money) if $K<S_{0}$ (resp. $K>S_{0}$ ), and finally they are said to be at-the-money if $K=S_{0}$.

2.2. Short maturity out-of-the-money Asian options. We will use large deviations theory to compute the leading order approximation at $T \rightarrow 0$ for the price of the out-ofthe-money Asian options.

Theorem 2. Assume that (2) and (3) both hold.

(i) For out-of-the-money call Asian options, i.e., $K>S_{0}$,

$$
C(T)=e^{-\frac{1}{T} \mathcal{I}\left(K, S_{0}\right)+o\left(\frac{1}{T}\right)}, \quad \text { as } T \rightarrow 0 .
$$

(ii) For out-of-the-money put Asian options, i.e., $K<S_{0}$,

$$
P(T)=e^{-\frac{1}{T} \mathcal{I}\left(K, S_{0}\right)+o\left(\frac{1}{T}\right)}, \quad \text { as } T \rightarrow 0 .
$$

where for any $S_{0}, K>0$,

$$
\mathcal{I}\left(K, S_{0}\right):=\inf _{\substack{\int_{0}^{1} e^{g(t)} d t=K \\ g(0)=\log S_{0}, g \in \mathcal{A C}[0,1]}} \frac{1}{2} \int_{0}^{1}\left(\frac{g^{\prime}(t)}{\sigma\left(e^{g(t)}\right)}\right)^{2} d t,
$$

where $\mathcal{A C}[0,1]$ is the space of absolutely continuous functions on $[0,1]$.

The variational problem in (13) will be solved completely in Proposition 8.

Remark 3. The small maturity asymptotics given by Theorem 2 and the rate function $\mathcal{I}\left(K, S_{0}\right)$ are independent of the interest rate $r$ and dividend yield $q$. These quantities contribute only to subleading order in the $T \rightarrow 0$ expansion. This is analogous to the wellknown BBF result for the small maturity European options in the local volatility model [4, which is also independent of $r, q$.

Remark 4. For at-the-money case, i.e. $K=S_{0}$, by letting $g(t) \equiv 0$ in $(13)$, we see that $\mathcal{I}\left(K, S_{0}\right)=0$. Indeed, Theorem 6 will give more precise asymptotics for at-the-money short maturity Asian options.

The asymptotics for short-maturity in-the-money Asian call and put options can be obtained as a corollary of the results for out-of-the-money case in Theorem 2 by a simple application of the put-call parity. 
Corollary 5. Assume that (2) and (3) both hold.

(i) For in-the-money call Asian options, i.e., $K<S_{0}$,

$$
C(T)=S_{0}-K-\frac{1}{2}(r+q) S_{0} T+K r T+O\left(T^{2}\right), \quad \text { as } T \rightarrow 0 .
$$

(ii) For in-the-money put Asian options, i.e., $K>S_{0}$,

$$
P(T)=K-S_{0}+\frac{1}{2}(r+q) S_{0} T-K r T+O\left(T^{2}\right), \quad \text { as } T \rightarrow 0 .
$$

2.3. At-the-Money Asian Options. When $K=S_{0}$, the Asian call and put options are at-the-money. We have the following result:

Theorem 6. Assume that the function $\sigma(s) s$ and $\sigma(s)$ are uniformly Lipschitz, i.e., there exist $\alpha, \beta>0$, such that for any $x, y \geq 0$,

$$
|\sigma(x) x-\sigma(y) y| \leq \alpha|x-y|, \quad|\sigma(x)-\sigma(y)| \leq \beta|x-y| .
$$

(i) When $K=S_{0}$, as $T \rightarrow 0$,

$$
C(T)=\sigma\left(S_{0}\right) S_{0} \frac{\sqrt{T}}{\sqrt{6 \pi}}+O(T) .
$$

(ii) When $K=S_{0}$, as $T \rightarrow 0$,

$$
P(T)=\sigma\left(S_{0}\right) S_{0} \frac{\sqrt{T}}{\sqrt{6 \pi}}+O(T) .
$$

Remark 7. Comparing Theorem [6 with Theorem 2, we see that the asymptotics for out-ofthe-money short maturity Asian options are governed by the rare events (large deviations), while the asymptotics for at-the-money short maturity Asian options are governed by the fluctuations about the typical events (Gaussian fluctuations).

\section{Variational Problem for Short-Maturity Asymptotics for Asian Options}

We present in this section the solution of the variational problem for the short-time asymptotics of the out-of-the-money Asian options given by Theorem 2. The solution is given by the following result.

Proposition 8. The rate function $\mathcal{I}\left(K, S_{0}\right)$ appearing in Theorem 2 is given by

$$
\mathcal{I}\left(K, S_{0}\right)= \begin{cases}\frac{1}{2} F^{(+)}\left(h_{1}\right) G^{(+)}\left(h_{1}\right) & K \leq S_{0}, \\ \frac{1}{2} F^{(-)}\left(f_{1}\right) G^{(-)}\left(f_{1}\right) & K \geq S_{0} .\end{cases}
$$

The two cases are as follows:

(i) $K \leq S_{0} . h_{1} \geq 0$ is the solution of the equation

$$
\frac{K}{S_{0}}-e^{-h_{1}}=\frac{G^{(+)}\left(h_{1}\right)}{F^{(+)}\left(h_{1}\right)},
$$

with

$$
\begin{aligned}
& G^{(+)}\left(h_{1}\right)=\int_{0}^{h_{1}} \frac{1}{\sigma\left(S_{0} e^{-y}\right)} \sqrt{e^{-y}-e^{-h_{1}}} d y, \\
& F^{(+)}\left(h_{1}\right)=\int_{0}^{h_{1}} \frac{1}{\sigma\left(S_{0} e^{-y}\right)} \frac{1}{\sqrt{e^{-y}-e^{-h_{1}}}} d y .
\end{aligned}
$$


(ii) $K \geq S_{0} \cdot f_{1} \geq 0$ is given by the solution of the equation

$$
e^{f_{1}}-\frac{K}{S_{0}}=\frac{G^{(-)}\left(f_{1}\right)}{F^{(-)}\left(f_{1}\right)}
$$

with

$$
\begin{aligned}
& G^{(-)}\left(f_{1}\right)=\int_{0}^{f_{1}} \frac{1}{\sigma\left(S_{0} e^{y}\right)} \sqrt{e^{f_{1}}-e^{y}} d y \\
& F^{(-)}\left(f_{1}\right)=\int_{0}^{f_{1}} \frac{1}{\sigma\left(S_{0} e^{y}\right)} \frac{1}{\sqrt{e^{f_{1}}-e^{y}}} d y .
\end{aligned}
$$

We would like to further study the properties of the rate function $\mathcal{I}\left(K, S_{0}\right)$. In particular, we will show that for a general local volatility model, the rate function $\mathcal{I}\left(K, S_{0}\right)$ is continuous in $K$ and it is increasing in $K$ for $K>S_{0}$ and decreasing in $K$ for $K<S_{0}$. This is based on an alternative representation of the rate function.

Proposition 9. (i) For $K>S_{0}$ the rate function $\mathcal{I}\left(K, S_{0}\right)$ is given by

$$
\mathcal{I}\left(K, S_{0}\right)=\inf _{\varphi>K / S_{0}} \frac{1}{2} \frac{\left(\mathcal{G}^{(-)}(\varphi)\right)^{2}}{\varphi-\frac{K}{S_{0}}},
$$

where we denoted

$$
\mathcal{G}^{(-)}(\varphi)=\int_{1}^{\varphi} \frac{\sqrt{\varphi-z}}{z \sigma\left(S_{0} z\right)} d z, \quad \varphi \geq 1
$$

(ii) for $K<S_{0}$ the rate function $\mathcal{I}\left(K, S_{0}\right)$ is given by

$$
\mathcal{I}\left(K, S_{0}\right)=\inf _{0<\chi<K / S_{0}} \frac{1}{2} \frac{\left(\mathcal{G}^{(+)}(\chi)\right)^{2}}{\frac{K}{S_{0}}-\chi},
$$

where we denoted

$$
\mathcal{G}^{(+)}(\chi)=\int_{\chi}^{1} \frac{\sqrt{z-\chi}}{z \sigma\left(S_{0} z\right)} d z, \quad 0<\chi \leq 1 .
$$

Remark 10. In the formulation in Proposition 9, we can see that the rate functions given in (26) and (28) are indeed continuous in the parameter $K$.

Using the result of Proposition 9 we can also prove that:

Proposition 11. The rate function $\mathcal{I}\left(K, S_{0}\right)$ is a monotonically increasing function of $K$ for $K>S_{0}$ and monotonically decreasing function of $K$ for $K<S_{0}$.

3.1. Black-Scholes Model. In the Black-Scholes model the volatility is constant $\sigma(S)=$ $\sigma$. The expression for the rate function $\mathcal{I}\left(K, S_{0}\right)$ simplifies and is given by the following result.

Proposition 12. The rate function for small maturity asymptotics for Asian options in the Black-Scholes model is

$$
\mathcal{I}_{\mathrm{BS}}\left(K, S_{0}\right)=\frac{1}{\sigma^{2}} \mathcal{J}_{\mathrm{BS}}\left(K / S_{0}\right)
$$



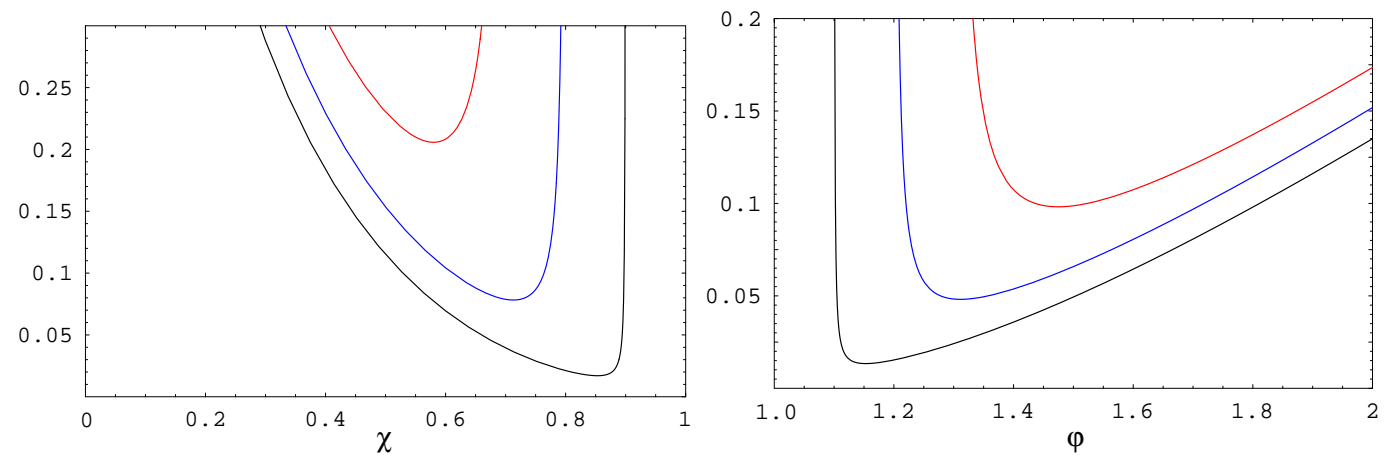

Figure 1. Plots of the functions in (26), (28) in the Black-Scholes model with $\sigma=1$ for $K / S_{0}=0.7,0.8,0.9$ (left) and $K / S_{0}=1.1,1.2,1.3$ (right). The infimum of the function gives the rate function in the BS model.

where $\mathcal{J}_{\mathrm{BS}}\left(K / S_{0}\right)$ depends only on the ratio $K / S_{0}$ and is given by

$$
\mathcal{J}_{\mathrm{BS}}\left(K / S_{0}\right)=\left\{\begin{array}{ll}
\frac{1}{2} \beta^{2}-\beta \tanh \left(\frac{\beta}{2}\right) & K \geq S_{0} \\
2 \xi(\tan \xi-\xi) & K \leq S_{0}
\end{array} .\right.
$$

For $K \geq S_{0}, \beta$ is the solution of the equation

$$
\frac{1}{\beta} \sinh \beta=\frac{K}{S_{0}},
$$

and for $K \leq S_{0}, \xi$ is the solution in the interval $\left[0, \frac{\pi}{2}\right]$ of the equation

$$
\frac{1}{2 \xi} \sin (2 \xi)=\frac{K}{S_{0}} \text {. }
$$

We note that the two equations $(32)$ and $(33)$ can be written in a common form as $\frac{1}{z} \sin z=K / S_{0}$ by denoting $z=2 \xi=i \beta$. With this notation, the rate function is $\mathcal{J}_{\mathrm{BS}}\left(K / S_{0}\right)=z \tan \left(\frac{1}{2} z\right)-\frac{1}{2} z^{2}$. We computed the function $\mathcal{J}_{\mathrm{BS}}\left(K / S_{0}\right)$ numerically, and the plot of this function is shown in Figure 2 .

We would like to find the series expansion of the Black-Scholes rate function $\mathcal{J}_{\mathrm{BS}}\left(K / S_{0}\right)$ around the ATM point $K / S_{0}=1$. The solutions of the equations for $\beta, \xi$ can be found by inverting the series in $k:=\frac{K}{S_{0}}-1$ for $(32)$ and $(33)$. Substituting into $\sqrt{31}$ we find the expansion of the rate function

$$
\mathcal{J}_{\mathrm{BS}}\left(\frac{K}{S_{0}}\right)=\frac{3}{2} k^{2}-\frac{9}{5} k^{3}+\frac{333}{175} k^{4}-\frac{1704}{875} k^{5}+O\left(k^{6}\right) .
$$

A similar expansion can be derived in powers of the $\log$-strike $x:=\log \left(K / S_{0}\right)$

$$
\mathcal{J}_{\mathrm{BS}}\left(\frac{K}{S_{0}}\right)=\frac{3}{2} x^{2}-\frac{3}{10} x^{3}+\frac{109}{1400} x^{4}-\frac{117}{7000} x^{5}+O\left(x^{6}\right) .
$$

Figure 2 (right plot) shows the approximations for the rate function $\mathcal{J}_{\mathrm{BS}}\left(K / S_{0}\right)$ obtained by keeping the first few terms in the series expansion (35). Keeping the first four terms in the expansion (35) matches the exact result for the rate function to an accuracy better than $1.2 \%$ for $x=\log \left(K / S_{0}\right) \in(-1.6,1.5)$. 

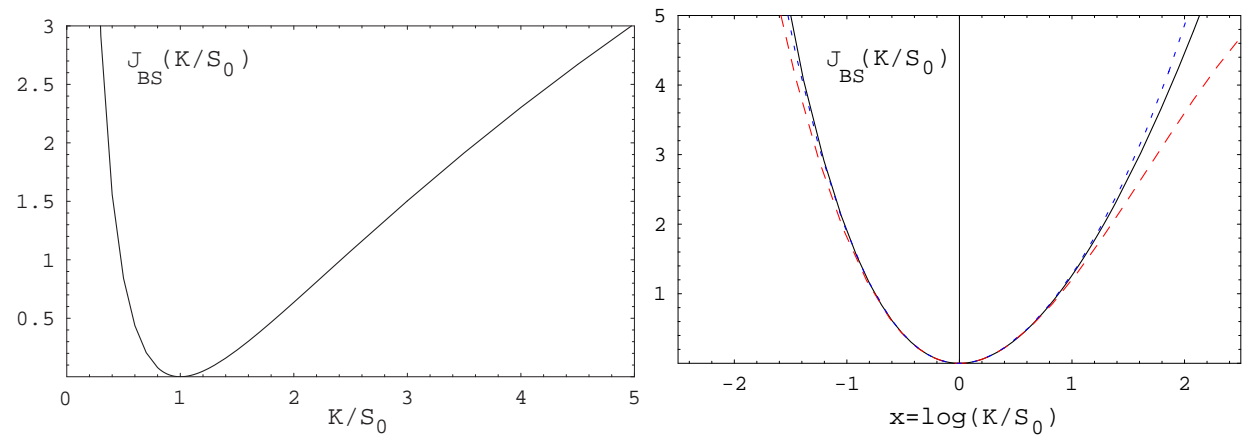

Figure 2. The rate function $\mathcal{J}_{\mathrm{BS}}\left(K / S_{0}\right)$ for the Asian options in the Black-Scholes model. This is related to $\mathcal{I}_{\mathrm{BS}}\left(K, S_{0}\right)$ as in (30). Left: $\mathcal{J}_{\mathrm{BS}}\left(K / S_{0}\right)$ vs. $K / S_{0}$. Right: the function $\mathcal{J}_{\mathrm{BS}}\left(K / S_{0}\right)$ vs. $x=\log \left(K / S_{0}\right)$ (black) and the Taylor series obtained by keeping terms up to $O\left(x^{3}\right)$ (dashed red) and $O\left(x^{4}\right)$ (dotted blue) in (35).

Next we consider the large/small-strike asymptotics of the rate function $\mathcal{J}_{\mathrm{BS}}\left(K / S_{0}\right)$. This is given by the following result.

Proposition 13. We have

$$
\mathcal{J}_{\mathrm{BS}}\left(\frac{K}{S_{0}}\right)= \begin{cases}\frac{1}{2} x^{2}+x \log (2 x)-x+3 \log ^{2}(2 x)-2 \log (2 x)+O\left(x^{-1}\right), & K \rightarrow \infty \\ 2 e^{-x}-2-\frac{\pi^{2}}{2}+O\left(e^{x}\right), & K \rightarrow 0\end{cases}
$$

with $x=\log \left(K / S_{0}\right)$ the log-strike.

3.2. General local volatility model. The rate function $\mathcal{I}\left(K, S_{0}\right)$ for the general local volatility function $\sigma(S)$ is given by Proposition 8. We give next an explicit result for the first three terms in the series expansion of $\mathcal{I}\left(K, S_{0}\right)$ in powers of $\log$-strike $x=\log \left(K / S_{0}\right)$.

Proposition 14. The first three terms in the series expansion of the rate function $\mathcal{I}\left(K, S_{0}\right)$ given by Proposition 8 in powers of log-strike $x=\log \left(K / S_{0}\right)$ are

$$
\begin{aligned}
\mathcal{I}\left(K, S_{0}\right)= & \frac{1}{b_{1}^{2}}\left\{\frac{3}{2} x^{2}+\left(-\frac{3}{10}-\frac{18}{5} \frac{b_{2}}{b_{1}^{2}}\right) x^{3}\right. \\
& \left.+\left(\frac{109}{1400}+\frac{117}{175} \frac{b_{2}}{b_{1}^{2}}+\frac{1872}{175} \frac{b_{2}^{2}}{b_{1}^{4}}-\frac{162}{35} \frac{b_{3}}{b_{1}^{3}}\right) x^{4}+O\left(x^{5}\right)\right\} .
\end{aligned}
$$

The coefficients $b_{i}$ depend on the local volatility function $\sigma(S)$, and are given by

$$
Y(z)=Z^{-1}(z)=\sum_{i=1}^{\infty} b_{i} z^{i},
$$

which is the inversion of the power series for the function

$$
Z(y)=\int_{0}^{y} \frac{d w}{\sigma\left(S_{0} e^{w}\right)}=\sum_{i=1}^{\infty} a_{i} y^{i}
$$

where we assumed that the local volatility function $\sigma(S)$ is sufficiently regular such that all the required derivatives exist and are finite. In particular the expansion (37) requires that $\sigma(S)$ is twice differentiable. 
Remark 15. For the Black-Scholes model we have $\sigma(S)=\sigma$ which gives $Z(y)=\frac{1}{\sigma} y$ and thus $Y(z)=\sigma z$. This gives $b_{1}=\sigma$ and $b_{j}=0$ for $j>1$. This recovers the expansion of the rate function in the Black-Scholes model given in equation (35).

Corollary 16. Assume that $\sigma(\cdot)$ is twice differentiable. The expansion (37) of the rate function $\mathcal{I}\left(K, S_{0}\right)$ for Asian options in the local volatility model (1) with local volatility function $\sigma(S)$ is given in a more explicit form as

$$
\begin{aligned}
& \mathcal{I}\left(K, S_{0}\right)=\frac{1}{\sigma^{2}\left(S_{0}\right)}\left\{\frac{3}{2} x^{2}+\left(-\frac{3}{10}-\frac{9}{5} S_{0} \frac{\sigma^{\prime}\left(S_{0}\right)}{\sigma\left(S_{0}\right)}\right) x^{3}\right. \\
& \left.+\left(\frac{109}{1400}-\frac{153}{350} S_{0} \frac{\sigma^{\prime}\left(S_{0}\right)}{\sigma\left(S_{0}\right)}+\frac{333}{175} S_{0}^{2}\left(\frac{\sigma^{\prime}\left(S_{0}\right)}{\sigma\left(S_{0}\right)}\right)^{2}-\frac{27}{35} S_{0}^{2} \frac{\sigma^{\prime \prime}\left(S_{0}\right)}{\sigma\left(S_{0}\right)}\right) x^{4}+O\left(x^{5}\right)\right\} .
\end{aligned}
$$

This is obtained by noting that the coefficients $a_{i}$ can be obtained by taking derivatives with respect to $y$ of $(39)$ at $y=0$. We get

$$
\begin{aligned}
& a_{1}=\frac{1}{\sigma\left(S_{0}\right)}, \\
& a_{2}=-\frac{1}{2} S_{0} \frac{\sigma^{\prime}\left(S_{0}\right)}{\sigma^{2}\left(S_{0}\right)}, \\
& a_{3}=-\frac{1}{6} S_{0} \frac{\sigma^{\prime}\left(S_{0}\right)}{\sigma^{2}\left(S_{0}\right)}+\frac{1}{3} S_{0}^{2} \frac{\left[\sigma^{\prime}\left(S_{0}\right)\right]^{2}}{\sigma^{3}\left(S_{0}\right)}-\frac{1}{6} S_{0}^{2} \frac{\sigma^{\prime \prime}\left(S_{0}\right)}{\sigma^{2}\left(S_{0}\right)} .
\end{aligned}
$$

Inverting the Taylor series (39) we find the coefficients $b_{i}$ :

$$
\begin{aligned}
& b_{1}=\frac{1}{a_{1}}=\sigma\left(S_{0}\right), \\
& b_{2}=-\frac{a_{2}}{a_{1}^{3}}=\frac{1}{2} S_{0} \sigma^{\prime}\left(S_{0}\right) \sigma\left(S_{0}\right), \\
& b_{3}=\frac{2 a_{2}^{2}}{a_{1}^{5}}-\frac{a_{3}}{a_{1}^{4}}=\frac{1}{6} S_{0}^{2}\left[\sigma^{\prime}\left(S_{0}\right)\right]^{2} \sigma\left(S_{0}\right)+\frac{1}{6} S_{0} \sigma^{\prime}\left(S_{0}\right) \sigma^{2}\left(S_{0}\right)+\frac{1}{6} S_{0}^{2} \sigma^{\prime \prime}\left(S_{0}\right) \sigma^{2}\left(S_{0}\right) .
\end{aligned}
$$

Substituting into (37) we obtain the result (40) for the rate function of the Asian option in the LV model to order $O\left(x^{3}\right)$, expressed only in terms of the ATM local volatility and its derivatives.

\section{Implied Volatility and Numerical Tests}

Implied volatility for European options was extensively studied in the mathematical finance literature. Due to the lack of a simple closed-form formula as in the Black-Scholes model for European options, the implied volatility for Asian options was much less studied. Our work on the short maturity asymptotics for the price of the Asian options for local volatility models can shed some light on the short maturity implied volatility for the Asian options.

4.1. Implied Volatility. The implied volatility $\sigma_{\text {implied }}$ is defined as the constant volatility which must be used in the Black-Scholes model for the Asian option such that its price matches that of the Asian option in the local volatility model. That is, we must have

$$
C_{B S}\left(K, S_{0}, \sigma_{\text {implied }}, T\right)=C\left(K, S_{0}, T\right)
$$


where $C\left(K, S_{0}, T\right)=e^{-r T} \mathbb{E}\left[\left(\frac{1}{T} \int_{0}^{T} S_{t} d t-K\right)^{+}\right]$, where $S_{t}$ satisfies the dynamics (1) with the local volatility $\sigma(\cdot)$ and $C_{B S}\left(K, S_{0}, \sigma_{\text {implied }}, T\right)=e^{-r t} \mathbb{E}\left[\left(\frac{1}{T} \int_{0}^{T} S_{t} d t-K\right)^{+}\right]$, where $S_{t}$ satisfies the dynamics (1) with $\sigma(\cdot) \equiv \sigma_{\text {implied }}$.

The fact that the implied volatility for an Asian option is well defined is not a trivial fact. It follows from the fact that the Vega of an Asian option in the Black-Scholes model is always positive, which was proved in Carr et al. [6], and hence, if one considers its price as a function of the volatility, the inverse of this function exists. As the volatility parameter $\sigma$ is increased from zero to infinity, the price of an out-ofthe-money Asian option goes from $C_{B S}\left(K, S_{0}, 0, T\right)=e^{-r T}\left(\frac{1}{T} \int_{0}^{T} S_{0} e^{(r-q) t} d t-K\right)^{+}$to $C_{B S}\left(K, S_{0}, \infty, T\right)=e^{-r T} \frac{1}{T} \int_{0}^{T} S_{0} e^{(r-q) t} d t$. Hence, the implied volatility $\sigma_{\text {implied }}$ is well defined. Note that it is trivial to see $C_{B S}\left(K, S_{0}, 0, T\right)=e^{-r T}\left(\frac{1}{T} \int_{0}^{T} S_{0} e^{(r-q) t} d t-K\right)^{+}$ but the statement $C_{B S}\left(K, S_{0}, \infty, T\right)=e^{-r T} \frac{1}{T} \int_{0}^{T} S_{0} e^{(r-q) t} d t$ is less trivial and we will give a rigorous proof of this statement in Proposition 32 in the Appendix.

¿From Theorem 2 for out-of-the-money Asian options, we can obtain the short-maturity limit of the implied volatility $\sigma_{\text {implied }}$. For simplicity, we only give a proof for the case $r=q=0$. We expect the same result holds for general $r, q$.

Proposition 17. Assume $r=q=0$ and (2) and (3) hold.

(i) The $T \rightarrow 0$ limit of the implied volatility of out-of-the-money Asian options in the local volatility model (1) is given by

$$
\lim _{T \rightarrow 0} \sigma_{\text {implied }}^{2}\left(K, S_{0}, T\right)=\frac{\mathcal{J}_{\mathrm{BS}}\left(K / S_{0}\right)}{\mathcal{I}\left(K, S_{0}\right)},
$$

where $\mathcal{I}\left(K, S_{0}\right)$ is given in Proposition 8 and $\mathcal{J}_{\mathrm{BS}}\left(K / S_{0}\right)$ is given in Proposition 12.

(ii) Under the same assumptions on $\sigma(\cdot)$ as in Theorem 6, the $T \rightarrow 0$ limit of the implied volatility of at-the-money Asian options in the local volatility model (1) is given by

$$
\lim _{T \rightarrow 0} \sigma_{\text {implied }}\left(K, S_{0}, T\right)=\sigma\left(S_{0}\right) .
$$

4.2. Equivalent Black-Scholes and Bachelier Volatility of Asian Options. One can define an equivalent Black-Scholes volatility of an Asian option, as that value of the volatility for which the Black-Scholes price of an European (vanilla) option with maturity $T$ and underlying value $A(T)$ reproduces the price of the Asian option with the same maturity $T$. We will denote this volatility as $\Sigma_{\mathrm{LN}}\left(K, S_{0}, T\right)$. We have thus

$$
\begin{aligned}
C\left(K, S_{0}, T\right) & =e^{-r T}\left[A(T) \Phi\left(d_{1}\right)-K \Phi\left(d_{2}\right)\right] \\
P\left(K, S_{0}, T\right) & =e^{-r T}\left[K \Phi\left(-d_{2}\right)-A(T) \Phi\left(-d_{1}\right)\right]
\end{aligned}
$$

where $A(T)$ is given in 99 and $d_{1,2}=\frac{1}{\Sigma_{L N} \sqrt{T}}\left(\log (A(T) / K) \pm \frac{1}{2} \Sigma_{L N}^{2} T\right)$. We remark that this is a natural definition since $A(T)$ is the forward price of the underlying of the Asian option $\mathbb{E}\left[\frac{1}{T} \int_{0}^{T} S_{t} d t\right]$. Using 50 ensures that put-call parity for Asian options 10 holds; this would not hold for example if one used $S_{0}$ instead of $A(T)$. The relation (50) also reproduces correctly the price of an Asian call option with zero strike $K=0: C\left(0, S_{0}, T\right)=$ $e^{-r T} \mathbb{E}\left[\frac{1}{T} \int_{0}^{T} S_{t} d t\right]=e^{-r T} A(T)$. A similar equivalent normal volatility $\Sigma_{\mathrm{N}}\left(K, S_{0}, T\right)$ of an Asian option can be defined in terms of the Bachelier option pricing formula. 
The equivalent log-normal volatility $\Sigma_{L N}$ defined as in (50) exists for any Asian call option price $C\left(K, S_{0}, T\right)$ satisfying the bounds $(A(T)-K)^{+} \leq e^{r T} C\left(K, S_{0}, T\right) \leq A(T)$ 43 . These bounds are indeed satisfied for Asian options under the local volatility model (1). The lower bound is satisfied by the convexity of the payoff $(x-K)^{+}$, and the upper bound follows from $(x-K)^{+} \leq x$.

Although Asian options are quoted in practice by price, and not implied volatility, such equivalent volatilities are a convenient representation of the short maturity asymptotics of the Asian option prices. Also, they give a natural formulation for the small-maturity asymptotics of the Asian options in Proposition 8, which is equivalent to a small-maturity limit for these volatilities. This result is somewhat similar to the representation of the small maturity asymptotics for European options in the local volatility model in terms of the BBF formula for the implied volatility [4].

The short maturity asymptotics for out-of-the-money Asian options given in Theorem 2 gives the following short-time asymptotics for the equivalent volatilities of the Asian options in the local volatility model (1). For simplicity, we only prove the $r=q=0$ case. We expect the same result holds for general $r, q$.

Proposition 18. Assume $r=q=0$ and (2) and (3) hold.

(i) The short-time limit $T \rightarrow 0$ of the Black-Scholes equivalent volatility of an out-ofthe-money Asian option is given by

$$
\lim _{T \rightarrow 0} \Sigma_{\mathrm{LN}}^{2}\left(K, S_{0}, T\right)=\frac{1}{2} \frac{\log ^{2}\left(\frac{K}{S_{0}}\right)}{\mathcal{I}\left(K, S_{0}\right)},
$$

and the corresponding result for the Bachelier equivalent volatility is

$$
\lim _{T \rightarrow 0} \Sigma_{\mathrm{N}}^{2}\left(K, S_{0}, T\right)=\frac{1}{2} \frac{\left(\frac{K}{S_{0}}-1\right)^{2}}{\mathcal{I}\left(K, S_{0}\right)},
$$

where $\mathcal{I}\left(K, S_{0}\right)$ is given in Proposition 8.

(ii) Under assumptions of Theorem 6 on $\sigma(\cdot)$, the short-time limit $T \rightarrow 0$ of the BlackScholes equivalent volatility of an at-the-money Asian option is given by

$$
\lim _{T \rightarrow 0} \Sigma_{\mathrm{LN}}\left(K, S_{0}, T\right)=\frac{1}{\sqrt{3}} \sigma\left(S_{0}\right),
$$

and the corresponding result for the Bachelier equivalent volatility is

$$
\lim _{T \rightarrow 0} \Sigma_{\mathrm{N}}\left(K, S_{0}, T\right)=\frac{1}{\sqrt{3}} \sigma\left(S_{0}\right) S_{0}
$$

In the Black-Scholes model we can get explicit results for the equivalent volatilities, using the result for the rate function $\mathcal{J}_{\mathrm{BS}}\left(K / S_{0}\right)$ derived in Proposition 12 . Denote the corresponding equivalent volatilities $\Sigma_{\mathrm{LN}}^{(B S)}$ and $\Sigma_{\mathrm{N}}^{(B S)}$. Their expansions about the ATM point in powers of $\log$-strike $x=\log \left(K / S_{0}\right)$ are

$$
\Sigma_{\mathrm{LN}}^{(B S)}\left(K / S_{0}\right)=\frac{1}{\sqrt{3}} \sigma\left(1+\frac{1}{10} x-\frac{23}{2100} x^{2}+\frac{1}{3500} x^{3}+O\left(x^{4}\right)\right),
$$

and in powers of $k=K / S_{0}-1$, respectively

$$
\Sigma_{\mathrm{N}}^{(B S)}\left(K / S_{0}\right)=\frac{1}{\sqrt{3}} \sigma S_{0}\left(1+\frac{3}{5} k-\frac{33}{350} k^{2}+\frac{83}{1750} k^{3}+O\left(k^{4}\right)\right) .
$$


Using the equation 40 we can derive similar results for a more general local volatility function $\sigma(S)$. From the first three terms in the Taylor expansion of the rate function in powers of log-strike we can get explicit results for the ATM equivalent volatility, the skew and smile convexity at the ATM point. We use $\Sigma_{\mathrm{LN}(\mathrm{N})}\left(K, S_{0}\right)$ to denote $\lim _{T \rightarrow 0} \Sigma_{\mathrm{LN}(\mathrm{N})}\left(K, S_{0}, T\right)$.

Proposition 19. Assume $\sigma(\cdot)$ is twice differentiable. The small maturity limit $T \rightarrow 0$ of the log-normal equivalent volatility for an Asian option in the local volatility model (1) has the expansion in $x=\log \left(K / S_{0}\right)$ around the ATM point

$$
\begin{aligned}
& \Sigma_{\mathrm{LN}}\left(K, S_{0}\right)=\frac{1}{\sqrt{3}} \sigma\left(S_{0}\right)\left\{1+\left(\frac{1}{10}+\frac{3}{5} S_{0} \frac{\sigma^{\prime}\left(S_{0}\right)}{\sigma\left(S_{0}\right)}\right) x\right. \\
& \left.+\left(-\frac{23}{2100}+\frac{57}{175} S_{0} \frac{\sigma^{\prime}\left(S_{0}\right)}{\sigma\left(S_{0}\right)}-\frac{33}{350} S_{0}^{2}\left(\frac{\sigma^{\prime}\left(S_{0}\right)}{\sigma\left(S_{0}\right)}\right)^{2}+\frac{9}{35} S_{0}^{2} \frac{\sigma^{\prime \prime}\left(S_{0}\right)}{\sigma\left(S_{0}\right)}\right) x^{2}+O\left(x^{3}\right)\right\} .
\end{aligned}
$$

The corresponding expansion for the normal equivalent volatility of an Asian option has the expansion in $k=\frac{K}{S_{0}}-1$ around the ATM point

$$
\begin{aligned}
& \Sigma_{\mathrm{N}}\left(K, S_{0}\right)=\frac{1}{\sqrt{3}} S_{0} \sigma\left(S_{0}\right)\left\{1+\left(\frac{3}{5}+\frac{3}{5} S_{0} \frac{\sigma^{\prime}\left(S_{0}\right)}{\sigma\left(S_{0}\right)}\right) k\right. \\
& \left.+\left(-\frac{33}{350}+\frac{57}{175} S_{0} \frac{\sigma^{\prime}\left(S_{0}\right)}{\sigma\left(S_{0}\right)}-\frac{33}{350} S_{0}^{2}\left(\frac{\sigma^{\prime}\left(S_{0}\right)}{\sigma\left(S_{0}\right)}\right)^{2}+\frac{9}{35} S_{0}^{2} \frac{\sigma^{\prime \prime}\left(S_{0}\right)}{\sigma\left(S_{0}\right)}\right) k^{2}+O\left(k^{3}\right)\right\} .
\end{aligned}
$$

The result of Proposition 19 is similar to the expansion of the implied volatility obtained in an uncorrelated local-stochastic volatility model 24] for the implied volatility of vanilla European options, see Theorem 4.1 in [24], giving the level, slope and convexity of the small-time implied volatility. Indeed, this result allows pricing Asian options directly from the observable European volatility skew, assuming local volatility dynamics, but without assuming a particular form of the local volatility function!

Combining the result of Proposition 19 and the well-known result for the ATM skew of European options in the local volatility model, see e.g. [24], we have:

Remark 20. In the short maturity limit,

$$
\text { ATM Asian vol }=\frac{1}{\sqrt{3}} \cdot \text { ATM European vol, }
$$

$$
\text { ATM Asian skew slope }=\frac{1}{\sqrt{3}} \cdot\left[\frac{1}{10} \cdot \text { ATM European vol }+\frac{6}{5} \cdot \text { ATM European skew }\right] \text {, }
$$

where the slope is with respect to the log-moneyness.

4.3. Numerical Tests. We present in this section a few numerical tests of the shortmaturity asymptotic results for Asian options obtained in this paper.

For ATM Asian options we have the result of Theorem 6 which can be used directly to obtain a price. For out-of-the-money Asian options, we have the asymptotic results of Theorem 2. In practice, we find that it is convenient to use this result to obtain the short-maturity equivalent log-normal volatility of an Asian option $\Sigma_{\mathrm{LN}}\left(K, S_{0}\right)$ (or normal volatility $\left.\Sigma_{\mathrm{N}}\left(K, S_{0}\right)\right)$, as given in Proposition 18 . 
TABLE 1. Numerical results for call (upper) and put (lower) Asian options with maturity $T=0.5,1,2$ years and parameters 61 obtained by MC simulation in the BS model (1 stdev in brackets), compared against the asymptotic results $C_{\text {as }}(K), P_{\text {as }}(K)$ given by (50). The last column gives the asymptotic equivalent log-normal volatility of the Asian option $\Sigma_{\mathrm{LN}}^{(B S)}$.

\begin{tabular}{|c|cc|cc|cc|c|}
\hline & \multicolumn{2}{|c|}{$T=0.5$} & \multicolumn{2}{c|}{$T=1$} & $T=2$ & \\
\hline$K$ & $\mathrm{MC}(\mathrm{n}=800)$ & $C_{\mathrm{as}}(K)$ & $\mathrm{MC}(\mathrm{n}=800)$ & $C_{\mathrm{as}}(K)$ & $\mathrm{MC}(\mathrm{n}=800)$ & $C_{\mathrm{as}}(K)$ & $\Sigma_{\mathrm{LN}}^{(B S)}$ \\
\hline \hline 100 & $4.8871(0.0078)$ & 4.8830 & $6.9037(0.0115)$ & 6.9013 & $9.7417(0.0155)$ & 9.7477 & $17.32 \%$ \\
105 & $2.9205(0.0062)$ & 2.9188 & $4.8848(0.0098)$ & 4.8847 & $7.7268(0.0155)$ & 7.7382 & $17.41 \%$ \\
110 & $1.6372(0.0046)$ & 1.6388 & $3.3689(0.0083)$ & 3.3715 & $6.0737(0.0139)$ & 6.0826 & $17.48 \%$ \\
115 & $0.8650(0.0033)$ & 0.8671 & $2.2698(0.0068)$ & 2.2745 & $4.7370(0.0124)$ & 4.7505 & $17.56 \%$ \\
120 & $0.4336(0.0023)$ & 0.4351 & $1.4980(0.0055)$ & 1.5033 & $3.6692(0.0110)$ & 3.6835 & $17.63 \%$ \\
125 & $0.2075(0.0016)$ & 0.2081 & $0.9715(0.0045)$ & 0.9758 & $2.8254(0.0098)$ & 2.8414 & $17.70 \%$ \\
130 & $0.0949(0.0011)$ & 0.0953 & $0.6201(0.0036)$ & 0.6234 & $2.1657(0.0086)$ & 2.1790 & $17.76 \%$ \\
\hline \hline$K$ & $\mathrm{MC}(\mathrm{n}=800)$ & $P_{\mathrm{as}}(K)$ & $\mathrm{MC}(\mathrm{n}=800)$ & $P_{\mathrm{as}}(K)$ & $\mathrm{MC}(\mathrm{n}=800)$ & $P_{\mathrm{as}}(K)$ & $\Sigma_{\mathrm{LN}}^{(B S)}$ \\
\hline \hline 70 & $0.0034(0.0001)$ & 0.0035 & $0.0810(0.0007)$ & 0.0809 & $0.5580(0.0024)$ & 0.5596 & $16.68 \%$ \\
75 & $0.0264(0.0004)$ & 0.0263 & $0.2579(0.0014)$ & 0.2580 & $1.1220(0.0036)$ & 1.1250 & $16.81 \%$ \\
80 & $0.1296(0.0009)$ & 0.1295 & $0.6608(0.0025)$ & 0.6609 & $2.0100(0.0050)$ & 2.0167 & $16.92 \%$ \\
85 & $0.4548(0.0018)$ & 0.4543 & $1.4221(0.0038)$ & 1.4237 & $3.2880(0.0067)$ & 3.2984 & $17.03 \%$ \\
90 & $1.2187(0.0031)$ & 1.2190 & $2.6671(0.0054)$ & 2.6711 & $4.9963(0.0085)$ & 5.0095 & $17.14 \%$ \\
95 & $2.6475(0.0048)$ & 2.6494 & $4.4820(0.0072)$ & 4.4877 & $7.1464(0.0103)$ & 7.1628 & $17.23 \%$ \\
100 & $4.8789(0.0066)$ & 4.8830 & $6.8928(0.0090)$ & 6.9013 & $9.7280(0.0121)$ & 9.7477 & $17.32 \%$ \\
\hline
\end{tabular}

The equivalent log-normal volatility $\Sigma_{\mathrm{LN}}$ given by Proposition 18 can be used in the Black-Scholes pricing formula as in (50) to compute Asian option prices. Although this approach introduces subleading terms in the option price which are not constrained by the asymptotic result of Theorem 2, we will demonstrate that it gives predictions that are in good agreement with the numerical simulations of the Asian option prices.

We consider next a few numerical tests of the asymptotic pricing formulas, on the example of the Asian options in the Black-Scholes model. One first test assumes the model parameters

$$
r=q=0, S_{0}=100, \sigma=30 \% .
$$

In Table 1 we show the prices of out-of-the-money call and put Asian options with maturities $T=0.5,1,2$ years, comparing the results of a Monte Carlo calculation against the asymptotic results $C_{\text {as }}$ and $P_{\text {as }}$ given in (50). The Monte Carlo simulation was performed with $N=10^{6}$ paths, and the time line was discretized with $n=800$ time steps. We note very good agreement of the short-maturity asymptotic results with the results of the Monte Carlo calculation. The asymptotic results are always within one standard deviation (68\% CL) of the MC result for $T=0.5,1$, and within two standard deviations (95\% CL) for $T=2$.

We also compare with the benchmark scenarios proposed in [27] and which were commonly used in the literature on pricing Asian options [11, 14, 39, 26, 50. We show in 
TABLE 2. Numerical results for Asian call options in the Black-Scholes model under the benchmark scenarios considered in [27, 39]. The last 4 columns show: a) the results from the asymptotic expansion (50) of this paper (PZ), b) the 3rd order approximation proposed in Foschi et al. [26] (FPP3), c) Levy approximation [38], d) precise evaluation using the spectral expansion in [39].

\begin{tabular}{ccccc|c|c|c|c}
\hline$r$ & $T$ & $S_{0}$ & $K$ & $\sigma$ & PZ & FPP3 & Levy & Linetsky \\
\hline \hline 0.02 & 1 & 2 & 2 & 0.1 & 0.055923 & 0.055986 & 0.056054 & 0.055986 \\
0.18 & 1 & 2 & 2 & 0.3 & 0.217054 & 0.218387 & 0.219829 & 0.218387 \\
0.0125 & 2 & 2 & 2 & 0.25 & 0.172163 & 0.172267 & 0.173490 & 0.172269 \\
0.05 & 1 & 1.9 & 2 & 0.5 & 0.192895 & 0.193164 & 0.195379 & 0.193174 \\
0.05 & 1 & 2 & 2 & 0.5 & 0.246125 & 0.246406 & 0.249791 & 0.246416 \\
0.05 & 1 & 2.1 & 2 & 0.5 & 0.305927 & 0.306210 & 0.310646 & 0.306220 \\
0.05 & 2 & 2 & 2 & 0.5 & 0.349314 & 0.350040 & 0.359204 & 0.350095 \\
\hline
\end{tabular}

Table 2 numerical results for the asymptotic approximation for the Asian options obtained from (50), for the scenarios proposed in [27]. They are compared against the very precise results of [39] obtained using a spectral expansion, and against the simple Levy approximation [38]. The asymptotic result performs in all cases better than the Levy approximation, and the agreement with the results of [39] is better than $0.7 \%$ in all cases.

The numerical tests presented show that the short-maturity asymptotic results of this paper can be used as a good approximation for Asian options prices with maturities relevant for practical applications.

\section{Asymptotics for Floating Strike Asian Options}

There are many variations of the standard Asian options in the finance literature and one of the most used is the so-called floating strike Asian options. The price of the floating strike Asian call/put options are given by

$$
\begin{aligned}
& C_{f}(T):=e^{-r T} \mathbb{E}\left[\left(\kappa S_{T}-\frac{1}{T} \int_{0}^{T} S_{t} d t\right)^{+}\right], \\
& P_{f}(T):=e^{-r T} \mathbb{E}\left[\left(\frac{1}{T} \int_{0}^{T} S_{t} d t-\kappa S_{T}\right)^{+}\right],
\end{aligned}
$$

where $\kappa>0$ is the strike, see e.g. [38, 41, 2, 9, 42, 35]. The floating-strike Asian option is more difficult to price than the fixed-strike case because the joint law of $S_{T}$ and $\frac{1}{T} \int_{0}^{T} S_{t} d t$ is needed, and also the one dimensional PDE that the floating-strike Asian price satisfies after a change of numéraire is difficult to solve numerically as the Dirac delta function appears as a coefficient, see e.g. [42, 2].

When $\kappa<1$, the call option is OTM, the put option is ITM; when $\kappa>1$, the call option is ITM, the put option is OTM; when $\kappa=1$, the call/put options are ATM. We are interested in the short maturity, i.e. $T \rightarrow 0$ asymptotics of these options.

For the Black-Scholes model, it was shown by Henderson and Wojakowski [35] that the floating-strike Asian options with continuous time averaging can be related to fixed strike 
ones as

$$
\begin{aligned}
& e^{-r T} \mathbb{E}\left[\left(\kappa S_{T}-A_{T}\right)^{+}\right]=e^{-q T} \mathbb{E}_{*}\left[\left(\kappa S_{0}-A_{T}\right)^{+}\right], \\
& e^{-r T} \mathbb{E}\left[\left(A_{T}-\kappa S_{T}\right)^{+}\right]=e^{-q T} \mathbb{E}_{*}\left[\left(A_{T}-\kappa S_{0}\right)^{+}\right] .
\end{aligned}
$$

The expectations on the right-hand side are taken with respect to a different measure $\mathbb{Q}_{*}$, where the asset price $S_{t}$ is given by the process

$$
d S_{t}=(q-r) S_{t} d t+\sigma S_{t} d W_{t}^{*},
$$

with $W_{t}^{*}$ a standard Brownian motion in the $\mathbb{Q}_{*}$ measure.

However, in our general setting of local volatility models, the equivalence relations (64) do not hold, and hence the asymptotics for floating strike Asian options must be obtained independently from that of the fixed strike Asian options. But it is not difficult to observe that the same techniques, i.e. large deviations, calculus of variation, Gaussian approximations, which were used to obtain the short-maturity asymptotics for the fixed strike Asian options, can be applied with little modification for the floating strike ones. For the sake of simplicity, we will only provide a sketch of the proofs in the Appendix.

Proposition 21. (i) Under the same assumptions as in Theorem 2, when $\kappa<1$,

$$
\begin{aligned}
& C_{f}(T)=e^{-\frac{1}{T} \mathcal{I}_{f}\left(\kappa, S_{0}\right)+o\left(\frac{1}{T}\right)}, \\
& P_{f}(T)=(1-\kappa) S_{0}-\frac{S_{0}}{2}(r+q) T+\kappa S_{0} q T+O\left(T^{2}\right),
\end{aligned}
$$

as $T \rightarrow 0$, where

$$
\mathcal{I}_{f}\left(\kappa, S_{0}\right)=\inf _{\substack{\int_{0}^{1} e^{g(t)} d t=\kappa e^{g(1)} \\ g(0)=\log S_{0}, g \in \mathcal{A C}[0,1]}} \frac{1}{2} \int_{0}^{1}\left(\frac{g^{\prime}(t)}{\sigma\left(e^{g(t)}\right)}\right)^{2} d t .
$$

(ii) Under the same assumptions as in Theorem 2, when $\kappa>1$,

$$
\begin{aligned}
& P_{f}(T)=e^{-\frac{1}{T} \mathcal{I}_{f}\left(\kappa, S_{0}\right)+o\left(\frac{1}{T}\right)}, \\
& C_{f}(T)=(\kappa-1) S_{0}+\frac{S_{0}}{2}(r+q) T-\kappa S_{0} q T+O\left(T^{2}\right),
\end{aligned}
$$

as $T \rightarrow 0$, where $\mathcal{I}_{f}$ is defined in (68).

(iii) Under the same assumptions as in Theorem 6, when $\kappa=1$,

$$
\lim _{T \rightarrow 0} \frac{1}{\sqrt{T}} C_{f}(T)=\lim _{T \rightarrow 0} \frac{1}{\sqrt{T}} P_{f}(T)=\frac{1}{\sqrt{6 \pi}} \sigma\left(S_{0}\right) S_{0} .
$$

For a general local volatility function $\sigma(S)$, the rate function $I_{f}\left(\kappa, S_{0}\right)$ is given by the following result:

Proposition 22. The solution of the variational problem in Proposition 21 for the short maturity asymptotics of floating strike Asian options is given by

$$
\mathcal{I}_{f}\left(\kappa, S_{0}\right)=\lambda(\kappa-1) e^{f_{1}}+\frac{1}{2} \lambda^{2} \kappa^{2} e^{2 f_{1}} \sigma^{2}\left(S_{0} e^{f_{1}}\right),
$$

where $f_{1}=f(1)$ with $f(t)$ given by the solution of the differential equation

$$
\frac{d}{d t}\left(\frac{f^{\prime}(t)}{\sigma\left(S_{0} e^{f(t)}\right)}\right)=\lambda e^{f(t)} \sigma\left(S_{0} e^{f(t)}\right)
$$


with

$$
\lambda=2 \frac{1-e^{f(1)}}{I_{s}[f]\left\{I_{s}[f]-2 \kappa e^{f_{1}} \sigma\left(S_{0} e^{f_{1}}\right)\right\}}, \quad I_{s}[f]=\int_{0}^{1} d s e^{f(s)} \sigma\left(S_{0} e^{f(s)}\right),
$$

and boundary conditions

$$
f(0)=0, \quad f^{\prime}(1)=\lambda \kappa e^{f_{1}} \sigma^{2}\left(S_{0} e^{f_{1}}\right) .
$$

We briefly outline in the following a possible numerical method for solving this calculus problem and finding the rate function $I_{f}\left(\kappa, S_{0}\right)$ for a given local volatility function $\sigma(S)$. This can be reduced to solving a non-linear equation for the variable $\lambda$. For a given value of $\lambda$, one can solve numerically the Euler-Lagrange equation (73) with the boundary conditions (75), and find the (non-optimal) function $f(t)$. Using this function we can compute the integral $I_{s}[f]$, and evaluate the expression for $\lambda$ in (74). Requiring that the result for $\lambda$ following from (74) is the same as the input value determines the value of this variable, and thus the optimal function $f(t)$. This equation for $\lambda$ can be solved by scanning over $\lambda$.

For the limiting case of the Black-Scholes model $\sigma(S)=\sigma$, the rate function $I_{f}\left(\kappa, S_{0}\right)$ can be found exactly, and is related to the Black-Scholes rate function $\mathcal{J}_{\mathrm{BS}}\left(K / S_{0}\right)$ given in Proposition 12 .

Proposition 23. In the Black-Scholes model, the rate function of the floating strike Asian option is given by

$$
\mathcal{I}_{f}(\kappa)=\inf _{\substack{\int_{0}^{1} e^{h(t)} d t=\kappa \\ h(0)=0, h \in \mathcal{A C}[0,1]}} \frac{1}{2 \sigma^{2}} \int_{0}^{1}\left(h^{\prime}(t)\right)^{2} d t=\frac{1}{\sigma^{2}} \mathcal{J}_{\mathrm{BS}}(\kappa),
$$

where $\mathcal{J}_{\mathrm{BS}}(\kappa)$ is the rate function for fixed strike Asian options in the Black-Scholes model given in 31.

Remark 24. Note that for the Black-Scholes model, $\mathcal{I}_{f}\left(\kappa, S_{0}\right)$ is independent of $S_{0}$ and hence we use the notation $\mathcal{I}_{f}(\kappa)$ in 76 .

Remark 25. The relation (76) is consistent with the equivalence relations (64). As noted above, the short maturity asymptotics of the Asian options is independent of the interest rate $r$ and dividend yield $q$ such that in this limit the expectations in the relation (64) are taken in the same measure. This implies that in the short maturity limit, fixed and floating strike Asian options are equal to each other, up to the substitution $K / S_{0} \mapsto \kappa$.

It is instructive to compare this explicit solution in the Black-Scholes model with the result of Proposition 22. For the case of constant volatility function $\sigma(S)=\sigma$, the relation (74) simplifies as

$$
\lambda_{\mathrm{BS}}(\kappa)=\frac{2}{\sigma^{2} \kappa^{2}} e^{-2 f(1)}\left(e^{f(1)}-1\right)=\frac{2}{\sigma^{2} \kappa^{2}} e^{2 h(1)}\left(e^{-h(1)}-1\right) .
$$

where $h(t)=f(1-t)-f(1)$ is the solution of the equivalent variational problem in 76 . This agrees with the solution for the Lagrange multiplier $\lambda$ for the auxiliary variational problem (76), which is given in (235). The value $\lambda_{\mathrm{BS}}(\kappa)$ (with $\left.\sigma=\sigma\left(S_{0}\right)\right)$ can be used as a starting point for scanning over the values of $\lambda$ in the numerical solution of the variational problem for the general local volatility model. 


\section{ApPEndix}

6.1. Large Deviation Principle and Contraction Principle. We start by giving a formal definition of the large deviation principle. We refer to Dembo and Zeitouni [12] or Varadhan [48] for general background of large deviations and the applications.

Definition 26 (Large Deviation Principle). A sequence $\left(P_{\epsilon}\right)_{\epsilon \in \mathbb{R}^{+}}$of probability measures on a topological space $X$ satisfies the large deviation principle with rate function $I: X \rightarrow \mathbb{R}$ if $I$ is non-negative, lower semicontinuous and for any measurable set $A$, we have

$$
-\inf _{x \in A^{o}} I(x) \leq \liminf _{\epsilon \rightarrow 0} \epsilon \log P_{\epsilon}(A) \leq \limsup _{\epsilon \rightarrow 0} \epsilon \log P_{\epsilon}(A) \leq-\inf _{x \in \bar{A}} I(x) .
$$

Here, $A^{o}$ is the interior of $A$ and $\bar{A}$ is its closure.

The contraction principle plays a key role in our proofs. For the convenience of the readers, we state the result as follows:

Theorem 27 (Contraction Principle, e.g. Theorem 4.2.1. [12]). If $P_{\epsilon}$ satisfies a large deviation principle on $X$ with rate function $I(x)$ and $F: X \rightarrow Y$ is a continuous map, then the probability measures $Q_{\epsilon}:=P_{\epsilon} F^{-1}$ satisfies a large deviation principle on $Y$ with rate function

$$
J(y)=\inf _{x: F(x)=y} I(x) .
$$

\subsection{Proofs of the Results in Section 2.}

Proof of Theorem 2. (i) We start by first proving the relation

$$
\lim _{T \rightarrow 0} T \log C(T)=\lim _{T \rightarrow 0} T \log \mathbb{P}\left(\frac{1}{T} \int_{0}^{T} S_{t} d t \geq K\right) .
$$

By Hölder's inequality, for any $\frac{1}{p}+\frac{1}{p^{\prime}}=1, p, p^{\prime}>1$,

$$
\begin{aligned}
C(T) & \leq e^{-r T} \mathbb{E}\left[\left|\frac{1}{T} \int_{0}^{T} S_{t} d t-K\right| 1_{\frac{1}{T} \int_{0}^{T} S_{t} d t \geq K}\right] \\
& \leq e^{-r T}\left(\mathbb{E}\left[\left|\frac{1}{T} \int_{0}^{T} S_{t} d t-K\right|^{p}\right]\right)^{\frac{1}{p}} \mathbb{P}\left(\frac{1}{T} \int_{0}^{T} S_{t} d t \geq K\right)^{\frac{1}{p^{\prime}}} .
\end{aligned}
$$

Let us assume that $p \geq 2$. Note that for $p \geq 2, x \mapsto x^{p}$ is a convex function for $x \geq 0$ and by Jensen's inequality, $\left(\frac{x+y}{2}\right)^{p} \leq \frac{x^{p}+y^{p}}{2}$ for any $x, y \geq 0$. Therefore

$$
\begin{aligned}
\mathbb{E}\left[\left|\frac{1}{T} \int_{0}^{T} S_{t} d t-K\right|^{p}\right] & \leq \mathbb{E}\left[\left(\frac{1}{T} \int_{0}^{T} S_{t} d t+K\right)^{p}\right] \\
& \leq 2^{p-1}\left[\mathbb{E}\left[\left(\frac{1}{T} \int_{0}^{T} S_{t} d t\right)^{p}\right]+K^{P}\right] .
\end{aligned}
$$

By Jensen's inequality again,

$$
\mathbb{E}\left[\left(\frac{1}{T} \int_{0}^{T} S_{t} d t\right)^{p}\right] \leq \mathbb{E}\left[\frac{1}{T} \int_{0}^{T} S_{t}^{p} d t\right]=\frac{1}{T} \int_{0}^{T} \mathbb{E}\left[S_{t}^{p}\right] d t .
$$


By Itô's formula,

$$
d\left(S_{t}^{p}\right)=p S_{t}^{p-1}\left[(r-q) S_{t} d t+\sigma\left(S_{t}\right) d W_{t}\right]+\frac{1}{2} p(p-1) S_{t}^{p-2} \sigma\left(S_{t}\right)^{2} S_{t}^{2} d t .
$$

Taking expectations of the both sides of the equation (85),

$$
d \mathbb{E}\left[S_{t}^{p}\right]=p(r-q) \mathbb{E}\left[S_{t}^{p}\right] d t+\frac{1}{2} p(p-1) \mathbb{E}\left[S_{t}^{p} \sigma\left(S_{t}\right)^{2}\right] d t .
$$

Since $\underline{\sigma} \leq \sigma(\cdot) \leq \bar{\sigma}$, we conclude that $\mathbb{E}\left[S_{t}^{p}\right] \leq m(t)$ where $m(t)$ is the solution to the ODE:

$$
d m(t)=p(r-q) m(t) d t+\frac{1}{2} p(p-1) \bar{\sigma}^{2} m(t) d t, \quad m(0)=S_{0}^{p},
$$

which has the solution $m(t)=S_{0}^{p} e^{\left(p(r-q)+\frac{1}{2} p(p-1) \bar{\sigma}^{2}\right) t}$. Hence,

$$
\frac{1}{T} \int_{0}^{T} \mathbb{E}\left[S_{t}^{p}\right] d t \leq \max _{0 \leq t \leq T} m(t) \leq S_{0}^{p} e^{\left|p(r-q)+\frac{1}{2} p(p-1) \bar{\sigma}^{2}\right| T} .
$$

Therefore, by equations (83), 84, 88), we have

$$
\limsup _{T \rightarrow 0} T \log C(T) \leq \limsup _{T \rightarrow 0} \frac{1}{p^{\prime}} T \log \mathbb{P}\left(\frac{1}{T} \int_{0}^{T} S_{t} d t \geq K\right) .
$$

Since it holds for any $2>p^{\prime}>1$, we have the upper bound.

For any $\epsilon>0$,

$$
\begin{aligned}
C(T) & \geq e^{-r T} \mathbb{E}\left[\left(\frac{1}{T} \int_{0}^{T} S_{t} d t-K\right) 1_{\frac{1}{T} \int_{0}^{T} S_{t} d t \geq K+\epsilon}\right] \\
& \geq e^{-r T} \epsilon \mathbb{P}\left(\frac{1}{T} \int_{0}^{T} S_{t} d t \geq K+\epsilon\right),
\end{aligned}
$$

which implies that

$$
\liminf _{T \rightarrow 0} T \log C(T) \geq \liminf _{T \rightarrow 0} T \log \mathbb{P}\left(\frac{1}{T} \int_{0}^{T} S_{t} d t \geq K+\epsilon\right) .
$$

Since it holds for any $\epsilon>0$, we get the lower bound.

So the problem boils down to compute the limit

$$
\lim _{T \rightarrow 0} T \log \mathbb{P}\left(\frac{1}{T} \int_{0}^{T} S_{t} d t \geq K\right)=\lim _{T \rightarrow 0} T \log \mathbb{P}\left(\int_{0}^{1} S_{t T} d t \geq K\right) .
$$

Let $X_{t}:=\log S_{t}$. This is equivalent to computing the limit

$$
\lim _{T \rightarrow 0} T \log \mathbb{P}\left(\int_{0}^{1} e^{X_{t T}} d t \geq K\right)
$$

where by Itô's lemma,

$$
d X_{t}=\left(r-q-\frac{1}{2} \sigma^{2}\left(e^{X_{t}}\right)\right) d t+\sigma\left(e^{X_{t}}\right) d W_{t}, \quad X_{0}=\log S_{0} .
$$

¿From the large deviations theory for small time diffusions, it was first proved in Varadhan [47. that under the assumptions (2), (3), $\mathbb{P}\left(X_{\cdot_{T}} \in \cdot\right)$ satisfies a sample path large deviation principle on $L_{\infty}[0,1]$ with the rate function

$$
I(g)=\frac{1}{2} \int_{0}^{1}\left(\frac{g^{\prime}(t)}{\sigma\left(e^{g(t)}\right)}\right)^{2} d t .
$$


with $g(0)=\log S_{0}$ and $g \in \mathcal{A C}[0,1]$, the space of absolutely continuous functions and $I(g)=+\infty$ otherwise.

Note that the map $g \mapsto \int_{0}^{1} e^{g(x)} d x$ from $L_{\infty}[0,1]$ to $\mathbb{R}^{+}$is a continuous map. Therefore, by contraction principle (Theorem 27$), \mathbb{P}\left(\int_{0}^{1} e^{X_{t T}} d t \in \cdot\right)$ satisfies a large deviation principle with the rate function

$$
\mathcal{I}\left(x, S_{0}\right):=\inf _{\substack{\int_{0}^{1} e^{g(t)} d t=x \\ g(0)=\log S_{0}, g \in \mathcal{A C}[0,1]}} \frac{1}{2} \int_{0}^{1}\left(\frac{g^{\prime}(t)}{\sigma\left(e^{g(t)}\right)}\right)^{2} d t .
$$

Hence, for out-of-the money call options, i.e. $S_{0}<K$,

$$
\lim _{T \rightarrow 0} T \log \mathbb{P}\left(\frac{1}{T} \int_{0}^{T} S_{t} d t \geq K\right)=-\inf _{x \geq K} \mathcal{I}\left(x, S_{0}\right)=-\mathcal{I}\left(K, S_{0}\right),
$$

where the last step is due to the fact that $\mathcal{I}\left(K, S_{0}\right)$ is increasing in $K$ for $K>S_{0}$, see Proposition 11.

(ii) We conclude by proving the analogous relation to 80 for Asian put options, that is, for out-of-the-money put options, $S_{0}>K$, we will show that

$$
\lim _{T \rightarrow 0} T \log \mathbb{P}\left(K \geq \frac{1}{T} \int_{0}^{T} S_{t} d t\right)=-\mathcal{I}\left(K, S_{0}\right) .
$$

By Hölder's inequality, for any $\frac{1}{p}+\frac{1}{p^{\prime}}=1, p, p^{\prime}>1$,

$$
\begin{aligned}
& P(T)=e^{-r T} \mathbb{E}\left[\left(K-\frac{1}{T} \int_{0}^{T} S_{t} d t\right)^{+} 1_{\left.K \geq \frac{1}{T} \int_{0}^{T} S_{t} d t\right]}\right. \\
& \leq e^{-r T}\left(\mathbb{E}\left[\left(\left(K-\frac{1}{T} \int_{0}^{T} S_{t} d t\right)^{+}\right)^{p}\right]\right)^{\frac{1}{p}} \mathbb{P}\left(K \geq \frac{1}{T} \int_{0}^{T} S_{t} d t\right)^{\frac{1}{p}} \\
& \leq e^{-r T} K \mathbb{P}\left(K \geq \frac{1}{T} \int_{0}^{T} S_{t} d t\right)^{\frac{1}{p^{\prime}}} \text {. }
\end{aligned}
$$

Thus, $\lim \sup _{T \rightarrow 0} T \log P(T) \leq-\frac{1}{p^{\prime}} \mathcal{I}\left(K, S_{0}\right)$. Since it holds for any $p^{\prime}>1$, we proved the upper bound.

For the lower bound, for any sufficiently small $\epsilon>0$,

$$
\begin{aligned}
P(T) & \geq e^{-r T} \mathbb{E}\left[\left(K-\frac{1}{T} \int_{0}^{T} S_{t} d t\right) 1_{\left.K \geq \frac{1}{T} \int_{0}^{T} S_{t} d t+\epsilon\right]}\right] \\
& \geq e^{-r T} \epsilon \mathbb{P}\left(K \geq \frac{1}{T} \int_{0}^{T} S_{t} d t+\epsilon\right),
\end{aligned}
$$

which implies that $\liminf _{T \rightarrow 0} T \log P(T) \geq-\mathcal{I}\left(K-\epsilon, S_{0}\right)$. By letting $\epsilon \downarrow 0$, and the fact that the rate function $\mathcal{I}\left(K, S_{0}\right)$ is continuous in $K$, see Proposition 9 and Remark 10 , we proved the lower bound. 
Proof of Corollary 5. (i) From the put-call parity,

$$
\begin{aligned}
C(T)-P(T) & =e^{-r T} \mathbb{E}\left[\frac{1}{T} \int_{0}^{T} S_{t} d t-K\right] \\
& =e^{-r T}\left[\frac{1}{T} \int_{0}^{T} e^{(r-q) t} S_{0} d t-K\right] \\
& = \begin{cases}e^{-r T}\left[S_{0}-K\right] & \text { if } r=q \\
e^{-r T}\left[\frac{1}{T(r-q)}\left[e^{(r-q) T}-1\right] S_{0}-K\right] & \text { if } r \neq q\end{cases} \\
& = \begin{cases}{\left[S_{0}-K\right](1-r T)+O\left(T^{2}\right)} & \text { if } r=q \\
S_{0}-K-\frac{1}{2}(r+q) S_{0} T+K r T+O\left(T^{2}\right) & \text { if } r \neq q\end{cases}
\end{aligned}
$$

as $T \rightarrow 0$. Therefore, for in-the-money call option, i.e. $S_{0}>K$, from Theorem 2 , we get $C(T)=S_{0}-K-\frac{1}{2}(r+q) S_{0} T+K r T+O\left(T^{2}\right)$ as $T \rightarrow 0$.

(ii) For in-the-money put option, i.e. $S_{0}<K$, from (i) and Theorem 2 , we get $P(T)=$ $K-S_{0}+\frac{1}{2}(r+q) S_{0} T-K r T+O\left(T^{2}\right)$ as $T \rightarrow 0$.

Proof of Theorem 6. (i) For at-the-money call option,

$$
C(T)=e^{-r T} \mathbb{E}\left[\left(\frac{1}{T} \int_{0}^{T} e^{(r-q) t} X_{t} d t-S_{0}\right)^{+}\right]
$$

where $X_{t}:=\frac{S_{t}}{e^{(r-q) t}}$ is a martingale and satisfies the SDE:

$$
d X_{t}=\sigma\left(X_{t} e^{(r-q) t}\right) X_{t} d W_{t},
$$

with $X_{0}=S_{0}$.

Claim 1. As $T \rightarrow 0$,

$$
\left|\mathbb{E}\left[\left(\frac{1}{T} \int_{0}^{T} e^{(r-q) t} X_{t} d t-S_{0}\right)^{+}\right]-\mathbb{E}\left[\left(\frac{1}{T} \int_{0}^{T} X_{t} d t-S_{0}\right)^{+}\right]\right|=O(T) .
$$

Let us prove (104).

$$
\begin{aligned}
& \left|\mathbb{E}\left[\left(\frac{1}{T} \int_{0}^{T} e^{(r-q) t} X_{t} d t-S_{0}\right)^{+}\right]-\mathbb{E}\left[\left(\frac{1}{T} \int_{0}^{T} X_{t} d t-S_{0}\right)^{+}\right]\right| \\
& \leq \mathbb{E}\left[\left|\left(\frac{1}{T} \int_{0}^{T} e^{(r-q) t} X_{t} d t-S_{0}\right)^{+}-\left(\frac{1}{T} \int_{0}^{T} X_{t} d t-S_{0}\right)^{+}\right|\right] \\
& \leq \mathbb{E}\left[\frac{1}{T} \int_{0}^{T}\left|e^{(r-q) t}-1\right| X_{t} d t\right] \\
& =S_{0} \frac{1}{T} \int_{0}^{T}\left|e^{(r-q) t}-1\right| d t \\
& =S_{0}\left|\frac{1}{T} \int_{0}^{T}\left(e^{(r-q) t}-1\right) d t\right| \\
& =S_{0}\left|\frac{e^{(r-q) T}-1}{(r-q) T}-1\right| .
\end{aligned}
$$


Hence, we proved (104).

Next, let us define $X_{t}$, which satisfies the SDE:

$$
d \hat{X}_{t}=\sigma\left(S_{0}\right) S_{0} d W_{t}, \quad \hat{X}_{0}=S_{0} .
$$

\section{Claim 2.}

$$
\mathbb{E}\left[\max _{0 \leq t \leq T}\left|X_{t}-\hat{X}_{t}\right|\right]=O(T), \quad \text { as } T \rightarrow 0
$$

Let us prove (107). Note that

$$
X_{t}-\hat{X}_{t}=\int_{0}^{t}\left[\sigma\left(X_{s} e^{(r-q) s}\right) X_{s}-\sigma\left(S_{0}\right) S_{0}\right] d W_{s}
$$

By Itô's isometry and the uniform Lipschitz assumption,

$$
\begin{aligned}
& \mathbb{E}\left[\left(X_{t}-\hat{X}_{t}\right)^{2}\right] \\
& =\int_{0}^{t} \mathbb{E}\left[\left(\sigma\left(X_{s} e^{(r-q) s}\right) X_{s}-\sigma\left(S_{0}\right) S_{0}\right)^{2}\right] d s \\
& \leq 2 \int_{0}^{t} \mathbb{E}\left[\left(\sigma\left(X_{s}\right) X_{s}-\sigma\left(S_{0}\right) S_{0}\right)^{2}\right] d s+2 \int_{0}^{t} \mathbb{E}\left[\left(\sigma\left(X_{s} e^{(r-q) s}\right) X_{s}-\sigma\left(X_{s}\right) X_{s}\right)^{2}\right] d s \\
& \leq 2 \alpha^{2} \int_{0}^{t} \mathbb{E}\left[\left(X_{s}-S_{0}\right)^{2}\right] d s+2 \beta^{2} \int_{0}^{t}\left(e^{(r-q) s}-1\right)^{2} \mathbb{E}\left[X_{s}^{4}\right] d s \\
& \leq 4 \alpha^{2} \int_{0}^{t} \mathbb{E}\left[\left(X_{s}-\hat{X}_{s}\right)^{2}\right] d s+4 \alpha^{2} \int_{0}^{t} \mathbb{E}\left[\left(\hat{X}_{s}-S_{0}\right)^{2}\right] d s+2 \beta^{2} \int_{0}^{t}\left(e^{(r-q) s}-1\right)^{2} \mathbb{E}\left[X_{s}^{4}\right] d s .
\end{aligned}
$$

Note that $\hat{X}_{s}=S_{0}+\sigma\left(S_{0}\right) S_{0} W_{s}$ and hence $\mathbb{E}\left[\left(\hat{X}_{s}-S_{0}\right)^{2}\right]=\sigma^{2}\left(S_{0}\right) S_{0}^{2} s$. Also

$$
|\sigma(x) x|=|\sigma(x) x-0 \sigma(0)| \leq \alpha|x|
$$

so that $\sigma(x) \leq \alpha$.

Since $X_{t}-X_{0}$ is a martingale starting at 0 , by the Burkholder-Davis-Gundy inequality, we get

$$
\mathbb{E}\left[\left(X_{t}-X_{0}\right)^{4}\right] \leq C \mathbb{E}\left[\left(\langle X\rangle_{t}\right)^{2}\right],
$$

for some constant $C>0$, where $\langle X\rangle_{t}=\int_{0}^{t} \sigma^{2}\left(X_{s} e^{(r-q) s}\right) X_{s}^{2} d s$ is the quadratic variation of $X_{t}$. Using the Cauchy-Schwarz inequality, we get

$$
\mathbb{E}\left[\left(X_{t}-X_{0}\right)^{4}\right] \leq C \mathbb{E}\left[\left(\int_{0}^{t} \sigma^{2}\left(X_{s} e^{(r-q) s}\right) X_{s}^{2} d s\right)^{2}\right] \leq C \mathbb{E}\left[\int_{0}^{t} \sigma^{4}\left(X_{s} e^{(r-q) s}\right) d s \int_{0}^{t} X_{s}^{4} d s\right] .
$$

Since $\sigma(\cdot) \leq \alpha$ and $\mathbb{E}\left[X_{t}^{4}\right] \leq 8 X_{0}^{4}+8 \mathbb{E}\left[\left(X_{t}-X_{0}\right)^{4}\right]\left(\right.$ since $\left(\frac{x+y}{2}\right)^{4} \leq \frac{x^{4}+y^{4}}{2}$ for any $\left.x, y \geq 0\right)$ and $X_{0}=S_{0}$, we get, for any sufficiently small $t$, say $t \leq 1$,

$$
\mathbb{E}\left[X_{t}^{4}\right] \leq 8 S_{0}^{4}+8 C \alpha^{4} \int_{0}^{t} \mathbb{E}\left[X_{s}^{4}\right] d s,
$$

Gronwall's inequality states that if $\beta(\cdot)$ is non-negative and for any $t \geq 0, u(t) \leq \alpha(t)+$ $\int_{0}^{t} \beta(s) u(s) d s$, then

$$
u(t) \leq \alpha(t)+\int_{0}^{t} \alpha(s) \beta(s) e^{\int_{s}^{t} \beta(r) d r} d s .
$$


Therefore, by Gronwall's inequality we have for any sufficiently small $t$,

$$
\mathbb{E}\left[X_{t}^{4}\right] \leq 8 S_{0}^{4} e^{8 C \alpha^{4} t}
$$

Hence, there exists a constant $\gamma>0$ so that

$$
2 \beta^{2} \int_{0}^{t}\left(e^{(r-q) s}-1\right)^{2} \mathbb{E}\left[X_{s}^{4}\right] d s \leq 16 \beta^{2} S_{0}^{4} e^{8 C \alpha^{4} t} \int_{0}^{t}\left(e^{(r-q) s}-1\right)^{2} d s \leq \gamma t^{2},
$$

for any sufficiently small $t>0$. Plugging into (109), we get

$$
\mathbb{E}\left[\left(X_{t}-\hat{X}_{t}\right)^{2}\right] \leq 4 \alpha^{2} \int_{0}^{t} \mathbb{E}\left[\left(X_{s}-\hat{X}_{s}\right)^{2}\right] d s+2 \alpha^{2} \sigma^{2}\left(S_{0}\right) S_{0}^{2} t^{2}+\gamma t^{2}
$$

By Gronwall's inequality, we have

$$
\begin{aligned}
\mathbb{E}\left[\left(X_{t}-\hat{X}_{t}\right)^{2}\right] & \leq\left[2 \alpha^{2} \sigma^{2}\left(S_{0}\right) S_{0}^{2}+\gamma\right] t^{2}+4 \alpha^{2} \int_{0}^{t}\left[2 \alpha^{2} \sigma^{2}\left(S_{0}\right) S_{0}^{2}+\gamma\right] s^{2} e^{4 \alpha^{2}(t-s)} d s \\
& \leq\left[2 \alpha^{2} \sigma^{2}\left(S_{0}\right) S_{0}^{2}+\gamma\right]\left[t^{2}+\frac{4}{3} \alpha^{2} t^{3} e^{4 \alpha^{2} t}\right] .
\end{aligned}
$$

Hence, we conclude that there exists some universal constant $M>0$, so that for any sufficiently small $T>0$,

$$
\mathbb{E}\left[\left(X_{T}-\hat{X}_{T}\right)^{2}\right] \leq M T^{2} .
$$

Finally notice that $X_{t}-\hat{X}_{t}$ is a martingale since both $X_{t}$ and $\hat{X}_{t}$ are. By Doob's martingale inequality, for sufficiently small $T>0$,

$$
\mathbb{E}\left[\max _{0 \leq t \leq T}\left|X_{t}-\hat{X}_{t}\right|\right] \leq 2\left(\mathbb{E}\left[\left(X_{T}-\hat{X}_{T}\right)^{2}\right]\right)^{\frac{1}{2}} \leq 2 \sqrt{M} T .
$$

Hence, we proved (107).

Claim 3. For $T \rightarrow 0$,

$$
\left|\mathbb{E}\left[\left(\frac{1}{T} \int_{0}^{T} X_{t} d t-S_{0}\right)^{+}\right]-\mathbb{E}\left[\left(\frac{1}{T} \int_{0}^{T} \hat{X}_{t} d t-S_{0}\right)^{+}\right]\right|=O(T) .
$$

Let us prove (117).

$$
\begin{aligned}
& \left|\mathbb{E}\left[\left(\frac{1}{T} \int_{0}^{T} X_{t} d t-S_{0}\right)^{+}\right]-\mathbb{E}\left[\left(\frac{1}{T} \int_{0}^{T} \hat{X}_{t} d t-S_{0}\right)^{+}\right]\right| \\
& \leq \mathbb{E}\left|\frac{1}{T} \int_{0}^{T} X_{t} d t-\frac{1}{T} \int_{0}^{T} \hat{X}_{t} d t\right| \\
& \leq \mathbb{E}\left[\max _{0 \leq t \leq T}\left|X_{t}-\hat{X}_{t}\right|\right] .
\end{aligned}
$$

Therefore, 117) follows from 107).

Claim 4.

$$
\mathbb{E}\left[\left(\frac{1}{T} \int_{0}^{T} \hat{X}_{t} d t-S_{0}\right)^{+}\right]=\sigma\left(S_{0}\right) S_{0} \frac{\sqrt{T}}{\sqrt{3}} \mathbb{E}\left[Z 1_{Z>0}\right]
$$


where $Z \sim N(0,1)$. Let us prove 119$)$. Note that $\hat{X}_{t}=S_{0}+\sigma\left(S_{0}\right) S_{0} W_{t}$. Hence,

$$
\frac{1}{T} \int_{0}^{T} \hat{X}_{t} d t-S_{0}=\sigma\left(S_{0}\right) S_{0} \frac{1}{T} \int_{0}^{T} W_{t} d t \sim N\left(0, \sigma^{2}\left(S_{0}\right) S_{0}^{2} \frac{T}{3}\right) .
$$

Hence, we proved 119 . Indeed, we can compute that

$$
\mathbb{E}\left[Z 1_{Z>0}\right]=\frac{1}{\sqrt{2 \pi}} \int_{0}^{\infty} x e^{-\frac{x^{2}}{2}} d x=\frac{1}{\sqrt{2 \pi}} .
$$

Finally, putting (104), (107), (117), (119), and (121) together, we proved the desired result.

(ii) For at-the-money put option, the proof is similar to (i) and is omitted.

\subsection{Proofs of the Results in Section 3 .}

Proof of Proposition 8. We present here the proof of the solution of the variational problem in Proposition 8 of the variational problem $(122)$. This variational problem can be simplified by introducing the function $f(t)$ defined as $g(t)=\log S_{0}+f(t)$. Expressed in terms of this function, the variational problem is stated as:

$$
\mathcal{I}\left(K, S_{0}\right)=\inf _{f} \frac{1}{2} \int_{0}^{1}\left(\frac{f^{\prime}(t)}{\sigma\left(S_{0} e^{f(t)}\right)}\right)^{2} d t
$$

where $f(t) \in \mathcal{A C}[0,1]$ satisfies $f(0)=0$ and

$$
\int_{0}^{1} e^{f(t)} d t=\frac{K}{S_{0}}
$$

The constraint (123) can be taken into account by introducing a Lagrange multiplier $\lambda$ and considering the variational problem for the functional

$$
\Lambda[f]=\frac{1}{2} \int_{0}^{1}\left(\frac{f^{\prime}(t)}{\sigma\left(S_{0} e^{f(t)}\right)}\right)^{2} d t+\lambda\left(\int_{0}^{1} e^{f(t)} d t-\frac{K}{S_{0}}\right) .
$$

with boundary condition $f(0)=0$.

First we recall a well-known result in the calculus of variations, see Sec. IV.5 in [10], stated in a form which is representative for the variational problems encountered in this paper.

Lemma 28. Consider the variational problem of finding the extremum of the functional

$$
\Lambda[x]=\frac{1}{2} \int_{0}^{T}\left(\frac{x^{\prime}(t)}{\Sigma(x(t))}\right)^{2} d t-\int_{0}^{T} V(x(t)) d t-f(x(T)),
$$

where $\Sigma(x), V(x)$ are $C^{1}$ functions, over the set of functions $x(t)$ satisfying the constraint

$$
x(0)=x_{0} .
$$

The optimal function $x(t)$ satisfies the Euler-Lagrange equation

$$
\frac{d}{d t}\left(\frac{x^{\prime}(t)}{\Sigma(x(t))}\right)=-V^{\prime}(x(t)) \Sigma(x(t))
$$

with boundary condition (126) at $t=0$ and the transversality condition

$$
x^{\prime}(T)=f(x(T)) \Sigma^{2}(x(T))
$$

at $t=T$. 
Proof. Define $x_{\epsilon}(t)$ as a perturbation around the optimal function $x(t)$

$$
x_{\epsilon}(t)=x(t)+\epsilon \eta(t),
$$

where $\eta(t) \in C^{1}$ is a function which vanishes at $t=0$ but is unconstrained at $t=T$

$$
\eta(0)=0 \text {. }
$$

A necessary condition that $\Lambda[x]$ has an extremum on $x(t)$ is that we have

$$
\begin{aligned}
\left.(131) \frac{d}{d \epsilon} \Lambda\left[x_{\epsilon}\right]\right|_{\epsilon=0}= & \int_{0}^{T} \frac{x^{\prime}(t)}{\Sigma^{2}(x(t))} \eta^{\prime}(t) d t \\
& -\int_{0}^{T}\left(\frac{\left[x^{\prime}(t)\right]^{2}}{\Sigma^{3}(x(t))} \Sigma^{\prime}(x(t))+V^{\prime}(x(t))\right) \eta(t) d t-f^{\prime}(x(T)) \eta(T) \\
= & \int_{0}^{T} \eta(t)\left\{-\frac{d}{d t}\left(\frac{x^{\prime}(t)}{\Sigma^{2}(x(t))}\right)-\frac{\left[x^{\prime}(t)\right]^{2}}{\Sigma^{2}(x(t))} \frac{\Sigma^{\prime}(x(t))}{\Sigma(x(t))}-V^{\prime}(x(t))\right\} d t \\
& +\eta(T)\left(\frac{x^{\prime}(T)}{\Sigma^{2}(x(T))}-f^{\prime}(x(T))\right)=0
\end{aligned}
$$

for any $\eta(t)$ satisfying the constraint 130 . In the second equality we integrated by parts in the first term. At optimality the functional derivative $\frac{d}{d \epsilon} \Lambda\left[x_{\epsilon}\right]$ must vanish for any $\eta(t)$ satisfying the constraint $\eta(0)=0$. This requires that the two terms vanish separately for any $\eta(t)$. The vanishing of the first term gives the Euler-Lagrange equation (127), and the vanishing of the second term gives the transversality condition (128).

Application of this result to the variational problem (124) gives that the optimal function $f$ satisfies the Euler-Lagrange equation

$$
\frac{d}{d t}\left(\frac{f^{\prime}(t)}{\sigma\left(S_{0} e^{f(t)}\right)}\right)=\lambda e^{f(t)} \sigma\left(S_{0} e^{f(t)}\right)
$$

and the transversality condition

$$
f^{\prime}(1)=0
$$

It is known [10] that one can relax the conditions $\Sigma(x), V(x) \in C^{1}$ to allow functions which are piecewise continuous. This is made explicit by writing the Euler-Lagrange equation $(132)$ in an alternative form as given by Lemma 29. Note that the derivative $\Sigma^{\prime}(x)$ does not appear in this result anymore. This implies that the conditions (2), (3) on $\sigma(\cdot)$ are sufficient for our purposes.

Lemma 29. The Euler-Lagrange equation (132) can be written alternatively as

$$
\frac{1}{2}\left(\frac{f^{\prime}(t)}{\sigma\left(S_{0} e^{f(t)}\right)}\right)^{2}-\lambda e^{f(t)}=c
$$

with $c$ a constant.

Proof. Follows by multiplying both sides of 132 with $f^{\prime}(t) / \sigma\left(S_{0} e^{f(t)}\right)$

$$
\frac{1}{2} \frac{d}{d t}\left(\frac{f^{\prime}(t)}{\sigma\left(S_{0} e^{f(t)}\right)}\right)^{2}=\lambda e^{f(t)} f^{\prime}(t)=\lambda \frac{d}{d t} e^{f(t)},
$$

This reproduces (134). 
¿From 134 and the transversality condition $f^{\prime}(1)=0$ we have

$$
\frac{1}{2}\left(\frac{f^{\prime}(t)}{\sigma\left(S_{0} e^{f(t)}\right)}\right)^{2}-\lambda e^{f(t)}=-\lambda e^{f(1)} .
$$

Integrating over $t:(0,1)$ this gives

$$
\mathcal{I}\left(K, S_{0}\right)=\frac{1}{2} \int_{0}^{1}\left(\frac{f^{\prime}(t)}{\sigma\left(S_{0} e^{f(t)}\right)}\right)^{2} d t=-\lambda e^{f(1)}+\lambda \int_{0}^{1} e^{f(t)} d t=\lambda\left(K / S_{0}-e^{f(1)}\right) .
$$

This relation expresses the rate function $\mathcal{I}\left(K, S_{0}\right)$ in terms of the terminal value of the optimal function $f(1)$ and the Lagrange multiplier $\lambda$.

The optimal function $f(t)$, the solution of the variational problem $(132)$, has the following qualitative behavior:

1. $f(t)$ is increasing $f^{\prime}(t)>0$ for $\lambda<0$. This case corresponds to $K>S_{0}$. For this case $f(1)>0$.

2. $f(t)$ is decreasing $f^{\prime}(t)<0$ for $\lambda>0$. This case corresponds to $K<S_{0}$. For this case $f(1)<0$.

These properties follow from the Euler-Lagrange equation $(132)$. Integrating this equation over $t:(t, 1)$ and using the transversality condition $f^{\prime}(1)=0$ gives

$$
f^{\prime}(t)=-\lambda \Sigma(f(t)) \int_{t}^{1} e^{f(s)} \Sigma(f(s)) d t .
$$

The factors multiplying $\lambda$ are negative, such that the sign of this expression is opposite to that of $\lambda$. The integrand in the constraint 123 satisfies the inequality $e^{f(t)}>e^{f(0)}=1$ in the case 1 . and $e^{f(t)}<e^{f(0)}=1$ in case 2 . These two cases correspond to $K>S_{0}$ and $K<S_{0}$, respectively.

We can use (134) to eliminate $f^{\prime}(t)$ in terms of $f(t)$ as

$$
f^{\prime}(t)= \begin{cases}\sqrt{-2 \lambda} \Sigma(f(t)) \sqrt{e^{f(1)}-e^{f(t)}}, & K>S_{0}, \lambda<0 \\ -\sqrt{2 \lambda} \Sigma(f(t)) \sqrt{e^{f(t)}-e^{f(1)}}, & K<S_{0}, \lambda>0\end{cases}
$$

We will treat the two cases separately.

Case 1. $K>S_{0}$. We will show that the rate function is

$$
\mathcal{I}\left(K, S_{0}\right)=\frac{1}{2} F^{(-)}\left(f_{1}\right) G^{(-)}\left(f_{1}\right),
$$

where $f_{1}>0$ is the solution of the equation

$$
e^{f_{1}}-K / S_{0}=\frac{G^{(-)}\left(f_{1}\right)}{F^{(-)}\left(f_{1}\right)},
$$

with

$$
\begin{aligned}
& G^{(-)}\left(f_{1}\right)=\int_{0}^{f_{1}} \frac{1}{\Sigma(y)} \sqrt{e^{f_{1}}-e^{y}} d y \\
& F^{(-)}\left(f_{1}\right)=\int_{0}^{f_{1}} \frac{1}{\Sigma(y)} \frac{1}{\sqrt{e^{f_{1}}-e^{y}}} d y .
\end{aligned}
$$

Proof. First we note that the rate function can be written in two equivalent ways as

$$
\mathcal{I}\left(K, S_{0}\right)=\lambda\left(K / S_{0}-e^{f_{1}}\right)=\sqrt{-\frac{\lambda}{2}} \int_{0}^{f_{1}} \frac{1}{\Sigma(y)} \sqrt{e^{f_{1}}-e^{y}} d y .
$$


The first equality is just equation (137) with $f_{1}=f(1)$. The second equality follows by changing the integration variable in the definition of the rate function from $t$ to $f(t)$

$$
\begin{aligned}
\mathcal{I}\left(K, S_{0}\right) & =\frac{1}{2} \int_{0}^{1}\left(\frac{f^{\prime}(t)}{\sigma\left(S_{0} e^{f(t)}\right)}\right)^{2} d t \\
& =\frac{1}{2} \int_{0}^{f_{1}} \frac{1}{f^{\prime}(t)}\left(\frac{f^{\prime}(t)}{\sigma\left(S_{0} e^{f(t)}\right)}\right)^{2} d f \\
& =\sqrt{-\frac{\lambda}{2}} \int_{0}^{f_{1}} \frac{1}{\Sigma(y)} \sqrt{e^{f_{1}}-e^{y}} d y \equiv \sqrt{-\frac{\lambda}{2}} G^{(-)}\left(f_{1}\right)
\end{aligned}
$$

where we used in the second line the relation $(139)$ to eliminate $f^{\prime}(t)$ in the numerator and denominator.

We need a second equation for $\left(\lambda, f_{1}\right)$ in order to be able to eliminate $\lambda$. This is obtained by writing

$$
\begin{aligned}
1 & =\int_{0}^{1} d t=\int_{0}^{f_{1}} \frac{1}{f^{\prime}(t)} d f \\
& =\frac{1}{\sqrt{-2 \lambda}} \int_{0}^{f_{1}} \frac{1}{\Sigma(y)} \frac{1}{\sqrt{e^{f_{1}}-e^{y}}} d y \equiv \frac{1}{\sqrt{-2 \lambda}} F^{(-)}\left(f_{1}\right) .
\end{aligned}
$$

Eliminating $\lambda$ between these equations we get the result (140).

Case 2. $K<S_{0}$. We will show that the rate function is

$$
\mathcal{I}\left(K, S_{0}\right)=\frac{1}{2} F^{(+)}\left(h_{1}\right) G^{(+)}\left(h_{1}\right),
$$

where $h_{1}=-f(1)>0$ is given by the solution of the equation

$$
\frac{K}{S_{0}}-e^{-h_{1}}=\frac{G^{(+)}\left(h_{1}\right)}{F^{(+)}\left(h_{1}\right)}
$$

with

$$
\begin{aligned}
& G^{(+)}\left(h_{1}\right)=\int_{0}^{h_{1}} \frac{1}{\Sigma(-y)} \sqrt{e^{-y}-e^{-h_{1}}} d y \\
& F^{(+)}\left(h_{1}\right)=\int_{0}^{h_{1}} \frac{1}{\Sigma(-y)} \frac{1}{\sqrt{e^{-y}-e^{-h_{1}}}} d y .
\end{aligned}
$$


Proof. The proof follows the same approach as in the previous case, using the relation (139) to eliminate $f^{\prime}(t)$. The rate function is

$$
\begin{aligned}
\mathcal{I}\left(K, S_{0}\right) & =\frac{1}{2} \int_{0}^{1}\left(\frac{f^{\prime}(t)}{\sigma\left(S_{0} e^{f(t)}\right)}\right)^{2} d t \\
& =\frac{1}{2} \int_{0}^{f_{1}} \frac{1}{f^{\prime}(t)}\left(\frac{f^{\prime}(t)}{\sigma\left(S_{0} e^{f(t)}\right)}\right)^{2} d f \\
& =-\sqrt{\frac{\lambda}{2}} \int_{0}^{f_{1}} \frac{1}{\Sigma(y)} \sqrt{e^{y}-e^{f_{1}}} d y \\
& =\sqrt{\frac{\lambda}{2}} \int_{0}^{h_{1}} \frac{1}{\Sigma(-y)} \sqrt{e^{-y}-e^{-h_{1}}} d y \\
& \equiv \sqrt{\frac{\lambda}{2}} G^{(+)}\left(h_{1}\right) .
\end{aligned}
$$

A second equation is derived in a similar way to 146 and reads

$$
\begin{aligned}
1 & =\int_{0}^{1} d t=\int_{0}^{f_{1}} \frac{1}{f^{\prime}(t)} d f=-\frac{1}{\sqrt{2 \lambda}} \int_{0}^{f_{1}} \frac{1}{\Sigma(y)} \frac{1}{\sqrt{e^{y}-e^{f_{1}}}} d y \\
& =\frac{1}{\sqrt{2 \lambda}} \int_{0}^{h_{1}} \frac{1}{\Sigma(-y)} \frac{1}{\sqrt{e^{-y}-e^{-h_{1}}}} d y \equiv \frac{1}{\sqrt{2 \lambda}} F^{(+)}\left(h_{1}\right) .
\end{aligned}
$$

Eliminating $\lambda$ between these equations we get the result (147). Equation (148) follows from (137) by writing

$$
\mathcal{I}\left(K, S_{0}\right)=\lambda\left(\frac{K}{S_{0}}-e^{-h_{1}}\right)=\frac{1}{2} F^{(+)}\left(h_{1}\right) G^{(+)}\left(h_{1}\right),
$$

and using $\lambda=\frac{1}{2}\left(F^{(+)}\left(h_{1}\right)\right)^{2}$.

Proof of Proposition 9. (i) $K>S_{0}$. The condition for the minimum of the function in (26) is

$$
\frac{d}{d \varphi} \frac{\left(\mathcal{G}^{(-)}(\varphi)\right)^{2}}{\varphi-\frac{K}{S_{0}}}=2 \mathcal{G}^{(-)}(\varphi) \frac{1}{2} \mathcal{F}^{(-)}(\varphi) \frac{1}{\varphi-\frac{K}{S_{0}}}-\left(\mathcal{G}^{(-)}(\varphi)\right)^{2} \frac{1}{\left(\varphi-\frac{K}{S_{0}}\right)^{2}}=0
$$

where we introduced

$$
\frac{d}{d \varphi} \mathcal{G}^{(-)}(\varphi)=\frac{1}{2} \mathcal{F}^{(-)}(\varphi)
$$

with

$$
\mathcal{F}^{(-)}(\varphi)=\int_{1}^{\varphi} \frac{1}{z \sigma\left(S_{0} z\right)} \frac{1}{\sqrt{\varphi-z}} d z, \quad \varphi \geq 1 .
$$

The equation (154) gives the value $\varphi_{1}$ at which the function has an extremal value

$$
\varphi_{1}-\frac{K}{S_{0}}=\frac{\mathcal{G}^{(-)}\left(\varphi_{1}\right)}{\mathcal{F}^{(-)}\left(\varphi_{1}\right)}
$$

This is identical to equation (23), with the identification $\varphi_{1}=e^{f_{1}}$, and $\mathcal{G}^{(-)}\left(\varphi_{1}\right)=$ $G^{(-)}\left(f_{1}\right), \mathcal{F}^{(-)}\left(\varphi_{1}\right)=F^{(-)}\left(f_{1}\right)$. 
Substituting the equation 157$)$ into $(26)$ we obtain the rate function

$$
\mathcal{I}\left(K, S_{0}\right)=\frac{1}{2} \mathcal{G}^{(-)}\left(\varphi_{1}\right) \mathcal{F}^{(-)}\left(\varphi_{1}\right)
$$

This agrees with the result (19) for the rate function for $K \geq S_{0}$.

(ii) $K<S_{0}$. The condition for the extremum of the function in 28 reads

$$
\frac{K}{S_{0}}-\chi_{1}=\frac{\mathcal{G}^{(+)}\left(\chi_{1}\right)}{\mathcal{F}^{(+)}\left(\chi_{1}\right)}
$$

where we defined

$$
\frac{d}{d \chi} \mathcal{G}^{(+)}(\chi)=-\frac{1}{2} \mathcal{F}^{(+)}(\chi)
$$

with

$$
\mathcal{F}^{(+)}(\chi)=\int_{\chi}^{1} \frac{1}{z \sigma\left(S_{0} z\right)} \frac{1}{\sqrt{z-\chi}} d z, \quad 0<\chi \leq 1 .
$$

The equation $(159)$ is the same as the equation $(20)$, with the identification $\chi_{1}=e^{-h_{1}}$, and $\mathcal{G}^{(+)}\left(\chi_{1}\right)=G^{(+)}\left(h_{1}\right), \mathcal{F}^{(+)}\left(\chi_{1}\right)=F^{(+)}\left(h_{1}\right)$. Substituting this equation into 28) we obtain the rate function as

$$
\mathcal{I}\left(K, S_{0}\right)=\frac{1}{2} \mathcal{G}^{(+)}\left(\chi_{1}\right) \mathcal{F}^{(+)}\left(\chi_{1}\right),
$$

which agrees with the result in (19) for $K \leq S_{0}$.

Proof of Proposition 11. The proof is based on Lemma 30 and Lemma 31.

i) $K>S_{0}$. We will use the representation 26 to show that the rate function $\mathcal{I}\left(K, S_{0}\right)$ is a monotonically increasing function of $K$ for $K>S_{0}$. First we prove that the function $\left(\mathcal{G}^{(-)}(\varphi)\right)^{2}$ satisfies the technical conditions required for $f(x)$ appearing in Lemma 30 , it is positive and increasing for $\varphi>1$, and has superlinear growth in $\varphi$ as $\varphi \rightarrow \infty$. The positivity condition follows from the definition, and the increasing property follows from the positivity of the integral $\mathcal{F}^{(-)}(\varphi)$ defined by $(156)$.

We prove next the superlinear growth condition as $\varphi \rightarrow \infty$. In the Black-Scholes model the function $\mathcal{G}^{(-)}(\varphi)$ can be computed exactly and is given by

$$
\mathcal{G}_{\mathrm{BS}}^{(-)}(\varphi)=\frac{1}{\sigma} \int_{1}^{\varphi} \frac{1}{z} \sqrt{\varphi-z} d z=\frac{2}{\sigma}\left(\sqrt{\varphi} \operatorname{arctanh}\left(\sqrt{\frac{\varphi-1}{\varphi}}\right)-\sqrt{\varphi-1}\right) .
$$

For $\varphi \rightarrow \infty$ this has the asymptotic expression

$$
\mathcal{G}_{\mathrm{BS}}^{(-)}(\varphi)=\frac{1}{\sigma}(\sqrt{\varphi} \log (4 \varphi)-2 \sqrt{\varphi}+O(1)), \quad \varphi \rightarrow \infty .
$$

We used here the asymptotic expansion of the arctanh function of large argument

$$
\operatorname{arctanh}(1-x)=-\frac{1}{2} \log (x / 2)+O(1), \quad x \rightarrow 0_{+} .
$$

The asymptotic expansion 164 shows that $\left(\mathcal{G}_{\mathrm{BS}}^{(-)}(\varphi)\right)^{2}$ grows faster than linear as $\varphi \rightarrow \infty$. This ensures the existence of a finite solution $x_{*}$ to the equation $(173)$ in the BS model. These results hold also in the general local volatility model with local volatility function $\sigma(S) \leq \bar{\sigma}$ bounded from above, as this implies a lower bound on the function $\left(\mathcal{G}^{(-)}(\varphi)\right)^{2}$ which has the same growth properties as $\varphi \rightarrow \infty$ as those obtained above in the BS model. 
ii) $K<S_{0}$. Using the representation (28) for the rate function, we have from Lemma 31 that $\mathcal{I}\left(K, S_{0}\right)$ is a decreasing function of $K$ for $0<K<S_{0}$. The function $\left(\mathcal{G}^{(+)}(\chi)\right)^{2}$ satisfies the technical conditions for $f(x)$ in Lemma 31. positivity follows from its definition, and the decreasing property for $0<\chi<1$, follows from the positivity of the integral $\mathcal{F}^{(+)}(\chi)$ defined in $(161)$.

The applicability of Lemma 31 requires also that the infimum in 161) is not reached at the lower boundary. We start by considering first the Black-Scholes model, where the function $\mathcal{G}^{(+)}(\chi)$ can be computed exactly and is given by

$$
\mathcal{G}_{\mathrm{BS}}^{(+)}(\chi)=\frac{1}{\sigma} \int_{\chi}^{1} \frac{1}{z} \sqrt{z-\chi} d z=\frac{2}{\sigma}\left(\sqrt{1-\chi}-\sqrt{\chi} \arctan \left(\sqrt{\frac{1}{\chi}-1}\right)\right) .
$$

The function $\mathcal{F}_{\mathrm{BS}}^{(+)}(\chi)$ is given by

$$
\mathcal{F}_{\mathrm{BS}}^{(+)}(\chi)=-2 \frac{d}{d \chi} \mathcal{G}_{\mathrm{BS}}^{(+)}(\chi)=\frac{2}{\sigma \sqrt{\chi}} \arctan \sqrt{\frac{1}{\chi}-1} .
$$

This has the asymptotic expression as $\chi \rightarrow 0$

$$
\mathcal{F}_{\mathrm{BS}}^{(+)}(\chi)=\frac{\pi}{\sigma \sqrt{\chi}}+O(1), \quad \chi \rightarrow 0
$$

Thus we have

$$
\lim _{\chi \rightarrow 0} \frac{d}{d \chi}\left(\mathcal{G}_{\mathrm{BS}}^{(+)}(\chi)\right)^{2}=-\infty
$$

where we used $\lim _{\chi \rightarrow 0} \mathcal{G}_{\mathrm{BS}}^{(+)}(\chi)=\frac{2}{\sigma}<\infty$.

This implies that the function $\left(\mathcal{G}_{\mathrm{BS}}^{(+)}(\chi)\right)^{2}$ satisfies the conditions 182 required for $f(x)$ in Lemma 31, which are sufficient to ensure that the infimum is not reached at the lower boundary $a$. We conclude that the statement of Lemma 31 applies to the BS model. These results hold also in the general local volatility model with local volatility function $\underline{\sigma} \leq \sigma(S) \leq \bar{\sigma}$ bounded from below and above. The upper bound on $\sigma(S)$ implies a lower bound for $\mathcal{F}^{(+)}(\chi)$, and the lower bound on $\sigma(S)$ implies an upper bound for $\mathcal{G}^{(+)}(0)$. Taken together these conditions ensure that the result 169 holds also for the general local volatility model.

Lemma 30. Let $f(x)$ be a positive and increasing function $f(x)>0, f^{\prime}(x)>0$, and define

$$
F(z)=\inf _{x>z}\left(\frac{f(x)}{x-z}\right)
$$

Furthermore, assume that $f(x)$ grows faster than $x$ as $x \rightarrow \infty$. Then $F(z)$ is a positive and increasing function

$$
F(z)>0, \quad F^{\prime}(z)>0 .
$$

Proof. The condition for the extremum of the function appearing in the definition of $F(z)$ is

$$
\frac{f^{\prime}(x)}{x-z}-\frac{f(x)}{(x-z)^{2}}=0
$$


Denote the solution of this equation $x_{*}$. This is given explicitly as

$$
x_{*}-z=\frac{f\left(x_{*}\right)}{f^{\prime}\left(x_{*}\right)} .
$$

This equation will have a solution for $z<x_{*}<\infty$ if $f(x)$ grows faster than linear as $x \rightarrow \infty$. This ensures that the infimum in 170 is not reached at $x \rightarrow \infty$.

Substituting into 170 we get

$$
F(z)=f^{\prime}\left(x_{*}\right)>0
$$

where we used $f^{\prime}\left(x_{*}\right)>0$. In order to prove the $F^{\prime}(z)>0$ property, we compute the derivative with respect to $z$

$$
F^{\prime}(z)=f^{\prime \prime}\left(x_{*}\right) \frac{d x_{*}}{d z}=f^{\prime \prime}\left(x_{*}\right) \frac{\left(f^{\prime}\left(x_{*}\right)\right)^{2}}{f^{\prime \prime}\left(x_{*}\right) f\left(x_{*}\right)}>0 .
$$

The second equality is obtained by taking a derivative of 173 with respect to $z$

$$
\begin{aligned}
\frac{d x_{*}}{d z}-1 & =\frac{d}{d z} \frac{f\left(x_{*}\right)}{f^{\prime}\left(x_{*}\right)}=\frac{d}{d x}\left(\frac{f\left(x_{*}\right)}{f^{\prime}\left(x_{*}\right)}\right) \frac{d x_{*}}{d z} \\
& =\frac{\left(f^{\prime}\left(x_{*}\right)\right)^{2}-f\left(x_{*}\right) f^{\prime \prime}\left(x_{*}\right)}{\left(f^{\prime}\left(x_{*}\right)\right)^{2}} \frac{d x_{*}}{d z}
\end{aligned}
$$

which gives

$$
\frac{d x_{*}}{d z}=\frac{\left(f^{\prime}\left(x_{*}\right)\right)^{2}}{f^{\prime \prime}\left(x_{*}\right) f\left(x_{*}\right)} .
$$

The inequality (175) proves that $F(z)$ is a monotonically increasing function.

Note that we do not need a convexity condition on $f(x)$ to obtain the monotonicity of $F(z)$. If $f^{\prime \prime}(x)>0$ then we get additionally that $\frac{d x_{*}}{d z}>0$, but this is not required for the monotonicity of $F(z)$.

Lemma 31. Let $f(x):[a, b] \rightarrow \mathbb{R}$ be a positive and decreasing function $f(x)>0, f^{\prime}(x)<$ 0 , and define

$$
F(z)=\inf _{x<z}\left(\frac{f(x)}{z-x}\right)
$$

Assuming that the infimum is not achieved on the boundary at $x=a$, then $F(z)$ is a positive and decreasing function

$$
F(z)>0, \quad F^{\prime}(z)<0, \quad a<z \leq b .
$$

Proof. The condition for the extremum of the function appearing in the definition of $F(z)$ is

$$
\frac{f^{\prime}(x)}{z-x}+\frac{f(x)}{(z-x)^{2}}=0 .
$$

Denote the solution of this equation $x_{*}$. This is given explicitly as

$$
x_{*}-z=\frac{f\left(x_{*}\right)}{f^{\prime}\left(x_{*}\right)} .
$$


We will assume that this equation has a solution for $a<x_{*}<z$. One possible way to ensure that the infimum is not reached at $a$ is to require

$$
\lim _{x \rightarrow a} f(x)<\infty, \quad \lim _{x \rightarrow a} f^{\prime}(x)=-\infty .
$$

Substituting into (178) we get

$$
F(z)=-f^{\prime}\left(x_{*}\right)
$$

Let us prove the $F^{\prime}(z)<0$ property. We have

$$
F^{\prime}(z)=-f^{\prime \prime}\left(x_{*}\right) \frac{d x_{*}}{d z}=-f^{\prime \prime}\left(x_{*}\right) \frac{\left(f^{\prime}\left(x_{*}\right)\right)^{2}}{f^{\prime \prime}\left(x_{*}\right) f\left(x_{*}\right)}<0 .
$$

The second equality is obtained by taking a derivative of (181) with respect to $z$, which gives the same result as in the proof of Lemma 30

$$
\frac{d x_{*}}{d z}=\frac{\left(f^{\prime}\left(x_{*}\right)\right)^{2}}{f^{\prime \prime}\left(x_{*}\right) f\left(x_{*}\right)} .
$$

The inequality (184) proves that $F(z)$ is a monotonically decreasing function.

Proof of Proposition 12. The variational problem in Proposition 8 simplifies for the case of a constant local volatility function $\sigma(S)=\sigma$, corresponding to the Black-Scholes model. The rate function is given by

$$
\mathcal{J}_{\mathrm{BS}}\left(K / S_{0}\right)=\sigma^{2} \mathcal{I}_{\mathrm{BS}}\left(K, S_{0}\right)=\frac{1}{2} \int_{0}^{1}\left[f^{\prime}(t)\right]^{2} d t
$$

where $f(t)$ is the solution of the Euler-Lagrange equation (the constant $a$ is related as $a=\lambda \sigma^{2}$ to the Lagrage multiplier appearing in the proof of Proposition 8)

$$
f^{\prime \prime}(t)=a e^{f(t)}
$$

with boundary conditions

$$
f(0)=0, \quad f^{\prime}(1)=0 .
$$

The unknown constant $a$ is determined by the condition

$$
\int_{0}^{1} d t e^{f(t)}=\frac{K}{S_{0}}
$$

The solution of the differential equation (187) can be found exactly. Two independent solutions of this equation are

$$
\begin{aligned}
& f_{1}(x)=\beta x-2 \log \left(\frac{e^{\beta x}+\gamma}{1+\gamma}\right), \\
& f_{2}(x)=-2 \log |\cos (\xi x+\eta)|+2 \log |\cos \eta| .
\end{aligned}
$$

The solution $f_{1}(x)$ of the equation (187) was given in [33], where the same equation appears in the context of an optimal importance sampling problem for Asian options in continuous time in the Black-Scholes model.

The parameters in these functions are determined by the boundary conditions (188), and by requiring that the functions satisfy the Euler-Lagrange equation (187).

It is easy to check, by direct substitution of (190), 191) into (187), that these functions are solutions of the Euler-Lagrange equation. The boundary condition at $x=0$ is satisfied 
automatically. Matching the constant factor in the Euler-Lagrange equation and requiring that also the boundary condition at $x=1$ is satisfied, gives the following constraints.

For $f_{1}(x)$ we have the equations

$$
\begin{aligned}
& 2 \gamma \beta^{2}=-a(1+\gamma)^{2}, \\
& \gamma=e^{\beta},
\end{aligned}
$$

which determine $a, \gamma$ in terms of $\beta$.

For $f_{2}(x)$ we have

$$
\begin{aligned}
& 2 \xi^{2}=a \cos ^{2}|\eta|, \\
& 2 \xi \tan |\xi+\eta|=0,
\end{aligned}
$$

which give $\eta=-\xi+k \pi$ with $k \in \mathbb{Z}$, and determine $a$ in terms of $\xi$. The multiple solutions for $\eta$ give the same solution $f_{2}(x)$ as we have $\cos (\xi(x-1)+k \pi)=(-1)^{k} \cos (\xi(x-1))$.

The constants $\xi, \beta$ can be determined from the constraint (189). For the solution (190), this is

$$
\int_{0}^{1} e^{f_{1}(x)} d x=\frac{1+\gamma}{e^{\beta}+\gamma} \frac{e^{\beta}-1}{\beta}=\frac{1}{\beta} \sinh \beta=\frac{K}{S_{0}},
$$

which reproduces (32). This is an equation for $\beta$ which has solutions only for $K>S_{0}$.

For the solution (191) we obtain

$$
\int_{0}^{1} e^{f_{2}(x)} d x=\int_{0}^{1} \frac{\cos ^{2} \xi}{\cos ^{2}(\xi(x-1))} d x=\frac{1}{\xi} \cos ^{2} \xi \tan \xi=\frac{K}{S_{0}}, \quad 0 \leq \xi<\frac{\pi}{2} .
$$

For $|\xi| \geq \frac{\pi}{2}$ the integral is divergent. This reproduces (33), and gives an equation for $\xi$ which has solutions only for $K<S_{0}$. The solution must satisfy $\xi \in\left(-\frac{\pi}{2}, \frac{\pi}{2}\right)$. Note that if $\xi^{*}$ is a solution to (33) in $\left(0, \frac{\pi}{2}\right)$, then $-\xi^{*} \in\left(-\frac{\pi}{2}, 0\right)$ is also a solution to (33) which yields the same value of $\mathcal{J}\left(K / S_{0}\right)$. Therefore, without loss of generality, we can assume that $\xi \in\left(0, \frac{\pi}{2}\right)$ which has a unique solution to 33 .

In conclusion, the solution of the Euler-Lagrange equation (187) with the boundary conditions (188) and constraint 189 is

$$
f(x)=\left\{\begin{array}{ll}
\beta x-2 \log \left(\frac{e^{\beta x}+e^{\beta}}{1+e^{\beta}}\right) & K \geq S_{0} \\
\log \left(\frac{\cos ^{2} \xi}{\cos ^{2}(\xi(x-1))}\right) & K \leq S_{0}
\end{array},\right.
$$

where $\beta$ and $\xi$ are the solutions of the equations $(32)$ and (33), respectively.

Finally, the rate function $\mathcal{J}_{\mathrm{BS}}\left(K / S_{0}\right)$ is found by substituting the solution 198$)$ for $f(x)$ into the equation (186) and performing the integration. This reproduces the result (31), which concludes the proof of Proposition 12 .

Proof of Proposition 13. (1) $K \geq S_{0}$. For $K / S_{0} \rightarrow \infty$, the solution of the equation (32) approaches $\beta \rightarrow \infty$. This suggests writing this equation as

$$
e^{\beta}=(2 \beta) \frac{K}{S_{0}} \frac{1}{1-e^{-2 \beta}},
$$

or

$$
\beta=x+\log (2 \beta)-\log \left(1-e^{-2 \beta}\right)=x+\log (2 \beta)+O\left(e^{-2 \beta}\right),
$$


with $x=\log \left(K / S_{0}\right)$. This implies that $\beta=x+O(\log x)$ as $x \rightarrow \infty$, and we can improve this estimate by iteration of (200), starting with the first order approximation

$$
\beta^{(0)}=x+o(x) .
$$

By substitution into 200 we get the successive iterations

$$
\begin{aligned}
\beta^{(1)} & =x+\log (2 x)+O\left(e^{-2 x}\right), \\
\beta^{(2)} & =x+\log (2 x)+\log [2(x+\log (2 x))]+O\left(e^{-2 x}\right) \\
& =x+\log (2 x)+\log (2 x)+\log \left(1+\frac{\log (2 x)}{x}\right)+O\left(e^{-2 x}\right) \\
& =x+2 \log (2 x)+\frac{\log (2 x)}{x}+O\left(x^{-2}\right) .
\end{aligned}
$$

The asymptotic expansion of the rate function is obtained by substituting into (31), and expanding to the order shown. This gives the result (36).

(2) $K \leq S_{0}$. The parameter $\xi$ is obtained by solving the equation (33). It is clear that as $K / S_{0} \rightarrow 0$, we have $\xi \rightarrow \pi / 2$.

It is convenient to introduce $\zeta$ defined as $\xi=\frac{\pi}{2}-\zeta$ with $\zeta \rightarrow 0$ in the $K / S_{0} \rightarrow 0$ limit. This is given by the solution of the equation

$$
\sin (2 \zeta)=\frac{K}{S_{0}}(\pi-2 \zeta)
$$

This equation can be solved again by iteration starting with $\zeta^{(0)}=0$.

The first two iterations are

$$
\begin{aligned}
\zeta^{(1)} & =\frac{\pi}{2} \frac{K}{S_{0}} \\
\zeta^{(2)} & =\frac{\pi}{2} \frac{K}{S_{0}}\left(1-\frac{K}{S_{0}}\right)+O\left(\left(K / S_{0}\right)^{3}\right) .
\end{aligned}
$$

Finally, substituting into (31) we obtain (36).

Proof of Proposition 14. We present the proof on the case $K \geq S_{0}$, the case $K<S_{0}$ is treated in a similar way, and leads to the same final result (37).

For given $\log$-strike $x=\log \left(K / S_{0}\right) \geq 0$, one has to find $f_{1}$ by solving the equation (23), and the rate function is obtained by substituting this value for $f_{1}$ into (19). We note that as $x \downarrow 0$, we have $f_{1} \downarrow 0$. Therefore it is reasonable to look for a solution for $f_{1}$ by expanding in $x$ around the ATM point $x=0$.

It is convenient to introduce the auxiliary variable $z_{1}$ such that $f_{1}=Y\left(z_{1}\right)$, with $Y(z):=Z^{-1}(z)$ the inverse of the function $Z(y)=\int_{0}^{y} \frac{d w}{\sigma\left(S_{0} e^{w}\right)}$. From the definition it is clear that for $x$ small, $z_{1}$ is also small, and they both go to zero simultaneously. The rationale of introducing $z_{1}$ is to absorb the dependence on $\sigma(S)$ in $F^{(-)}\left(f_{1}\right), G^{(-)}\left(f_{1}\right)$ into a change of the integration variable.

The strategy of the proof will be:

Step 1. Express the equation (23) for $f_{1}$ as an equation for $z_{1}$, expanded to a given order in $z_{1}$.

Step 2. Invert the expansion obtained in Step 1 and derive an expansion for $z_{1}$ in powers of the $\log$-strike $x$ to a given order. 
Step 3. Express the result $(19)$ for the rate function $\mathcal{I}\left(K, S_{0}\right)$ for $K \geq S_{0}$ as a series in $z_{1}$. Further, use here the expansion for $z_{1}$ in powers of $x$ obtained in Step 2.

Step 1. We start by deriving the expansion of the functions $G^{(-)}\left(f_{1}\right)$ and $F^{(-)}\left(f_{1}\right)$ in powers of $z_{1}=Z\left(f_{1}\right)$. First we absorb the dependence on the local volatility function $\sigma(S)$ in the definitions of these functions into a new integration variable $z$ defined such that $d z=d y / \sigma\left(S_{0} e^{y}\right)$. This gives

$$
\begin{aligned}
& G^{(-)}\left(f_{1}\right)=\int_{0}^{f_{1}} \frac{1}{\sigma\left(S_{0} e^{y}\right)} \sqrt{e^{f_{1}}-e^{y}} d y=\int_{0}^{z_{1}} \sqrt{e^{Y\left(z_{1}\right)}-e^{Y(z)}} d z, \\
& F^{(-)}\left(f_{1}\right)=\int_{0}^{f_{1}} \frac{1}{\sigma\left(S_{0} e^{y}\right)} \frac{1}{\sqrt{e^{f_{1}}-e^{y}}} d y=\int_{0}^{z_{1}} \frac{d z}{\sqrt{e^{Y\left(z_{1}\right)}-e^{Y(z)}}},
\end{aligned}
$$

where $Y(z)$ is the inverse of the function $Z(y)=\int_{0}^{y} \frac{d w}{\sigma\left(S_{0} e^{w}\right)}$, and $z_{1}=Z\left(f_{1}\right)$.

Substituting the Taylor series of $Y(z)$ in $(38)$, expanding in $z, z_{1}$ to a given order, and evaluating the integrals, we get

$$
\begin{aligned}
G^{(-)}\left(f_{1}\right)= & \sqrt{b_{1}} z_{1}^{3 / 2}\left(\frac{2}{3}+\frac{7}{30 b_{1}}\left(b_{1}^{2}+2 b_{2}\right) z_{1}\right. \\
& \left.+\frac{1}{1680 b_{1}^{2}}\left(81 b_{1}^{4}+628 b_{1}^{2} b_{2}-284 b_{2}^{2}+912 b_{1} b_{3}\right) z_{1}^{2}+O\left(z_{1}^{3}\right)\right), \\
F^{(-)}\left(f_{1}\right)= & \frac{1}{\sqrt{b_{1}}} \sqrt{z_{1}}\left(2-\frac{5}{6 b_{1}}\left(b_{1}^{2}+2 b_{2}\right) z_{1}\right. \\
& \left.+\frac{1}{240 b_{1}^{2}}\left(41 b_{1}^{4}-12 b_{1}^{2} b_{2}+516 b_{2}^{2}-528 b_{1} b_{3}\right) z_{1}^{2}+O\left(z_{1}^{3}\right)\right) .
\end{aligned}
$$

Next we express the equation (23) giving $f_{1}$ in terms of the $\log$-strike $x=\log \left(K / S_{0}\right)$, as an expansion in $z_{1}$. This is

$$
x=\log \left(e^{f_{1}}-\frac{G^{(-)}\left(f_{1}\right)}{F^{(-)}\left(f_{1}\right)}\right),
$$

Recalling that $f_{1}=Y\left(z_{1}\right)=b_{1} z_{1}+b_{2} z_{1}^{2}+\cdots$, we obtain by substitution on the right-hand side and Taylor expansion in powers of $z_{1}$

$$
\begin{aligned}
x= & \log \left(e^{Y\left(z_{1}\right)}-\frac{G^{(-)}\left(f_{1}\right)}{F^{(-)}\left(f_{1}\right)}\right) \\
= & \frac{2}{3} b_{1} z_{1}+\frac{1}{45}\left(b_{1}^{2}+22 b_{2}\right) z_{1}^{2} \\
& +\frac{1}{2835 b_{1}}\left(b_{1}^{4}+150 b_{1}^{2} b_{2}+48 b_{2}^{2}+1026 b_{1} b_{3}\right) z_{1}^{3}+O\left(z_{1}^{4}\right) .
\end{aligned}
$$

Step 2. Next we invert the series $(212)$ to get $z_{1}$ as an expansion in $x$

$$
\begin{aligned}
z_{1} & =\frac{3}{2 b_{1}} x+\left(-\frac{3}{40 b_{1}}-\frac{33 b_{2}}{20 b_{1}^{3}}\right) x^{2} \\
& +\frac{1}{1400 b_{1}^{5}}\left(8 b_{1}^{4}+87 b_{1}^{2} b_{2}+4962 b_{2}^{2}-2565 b_{1} b_{3}\right) x^{3}+O\left(x^{4}\right) .
\end{aligned}
$$


Step 3. Finally, we insert this expansion into the expression for the rate function (19), which gives the expansion of the rate function in powers of $x$

$$
\begin{aligned}
\mathcal{I}\left(K, S_{0}\right)= & \frac{1}{2} F^{(-)}\left(f_{1}\right) G^{(-)}\left(f_{1}\right)=\frac{3}{2 b_{1}^{2}} x^{2}-\frac{3}{10 b_{1}^{4}}\left(b_{1}^{2}+12 b_{2}\right) x^{3} \\
& +\frac{1}{1400 b_{1}^{6}}\left(109 b_{1}^{4}+936 b_{1}^{2} b_{2}+14976 b_{2}^{2}-6480 b_{1} b_{3}\right) x^{4}+O\left(x^{5}\right) .
\end{aligned}
$$

This reproduces the result (37).

Proposition 32. For any fixed $K, S_{0}, T$, the price of an Asian option in the Black-Scholes model approaches the following value in the infinite volatility limit

$$
\lim _{\sigma \rightarrow \infty} C_{B S}\left(K, S_{0}, \sigma, T\right)=e^{-r T} \frac{1}{T} \int_{0}^{T} S_{0} e^{(r-q) t} d t .
$$

Proof. For any $\epsilon>0$,

$$
\begin{aligned}
& \left|C_{B S}\left(K, S_{0}, \sigma, T\right)-e^{-r T} \mathbb{E}\left[\left(\frac{1}{T} \int_{\epsilon}^{T} S_{0} e^{(r-q) t+\sigma W_{t}-\frac{1}{2} \sigma^{2} t} d t-K\right)^{+}\right]\right| \\
& \leq e^{-r T} \mathbb{E}\left[\frac{1}{T} \int_{0}^{\epsilon} S_{0} e^{(r-q) t+\sigma W_{t}-\frac{1}{2} \sigma^{2} t} d t\right]=e^{-r T} \frac{1}{T} \int_{0}^{\epsilon} S_{0} e^{(r-q) t} d t,
\end{aligned}
$$

where the last term is independent of $\sigma$ and it goes to 0 as $\epsilon \rightarrow 0$. On the other hand, for almost every sample path, the Brownian motion $W_{t}$ is continuous in $t$ and therefore $\sup _{0 \leq t \leq T} W_{t}<\infty$. It follows that

$$
\frac{1}{T} \int_{\epsilon}^{T} S_{0} e^{(r-q) t+\sigma W_{t}-\frac{1}{2} \sigma^{2} t} d t \leq e^{\sigma \sup _{0 \leq t \leq T} W_{t}-\frac{1}{2} \sigma^{2} \epsilon} \frac{1}{T} \int_{\epsilon}^{T} S_{0} e^{(r-q) t} d t \rightarrow 0,
$$

a.s. as $\sigma \rightarrow \infty$. By bounded convergence theorem,

$$
\lim _{\sigma \rightarrow \infty} \mathbb{E}\left[\left(K-\frac{1}{T} \int_{\epsilon}^{T} S_{0} e^{(r-q) t+\sigma W_{t}-\frac{1}{2} \sigma^{2} t} d t\right)^{+}\right]=K .
$$

¿From put-call parity (Remark 1),

$$
\lim _{\sigma \rightarrow \infty} \mathbb{E}\left[\left(\frac{1}{T} \int_{\epsilon}^{T} S_{0} e^{(r-q) t+\sigma W_{t}-\frac{1}{2} \sigma^{2} t} d t-K\right)^{+}\right]=\frac{1}{T} \int_{\epsilon}^{T} S_{0} e^{(r-q) t} d t
$$

Finally, we let $\epsilon \rightarrow 0$ to complete the proof.

Proof of Proposition 17. (i) For the Black-Scholes model with $r=q=0$, the stock price follows a geometric Brownian motion, i.e., $S_{t}=S_{0} e^{\sigma W_{t}-\frac{1}{2} \sigma^{2} t}$. The price of the Asian call option in the Black-Scholes model is

$$
C_{A}^{(B S)}\left(S_{0}, K, \sigma, T\right)=\mathbb{E}\left[\left(S_{0} \int_{0}^{1} e^{\sigma W_{t T}-\frac{1}{2} \sigma^{2} t T} d t-K\right)^{+}\right] .
$$

¿From the Brownian scaling property,

$$
C_{A}^{(B S)}\left(S_{0}, K, \sigma, T\right)=\mathbb{E}\left[\left(S_{0} \int_{0}^{1} e^{\sigma \sqrt{T}\left(W_{t T} / \sqrt{T}\right)-\frac{1}{2}\left(\sigma^{2} T\right) t} d t-K\right)^{+}\right]:=\tilde{C}_{A}^{(B S)}\left(S_{0}, K, \sigma^{2} T\right)
$$


can be viewed as a function of $\sigma^{2} T$. Moreover the result in Carr et al. [6] implies that $\tilde{C}_{A}^{(B S)}\left(S_{0}, K, \sigma^{2} T\right)$ is increasing as a function of $\sigma^{2} T$.

Consider first an out-of-the-money Asian call option $K>S_{0}$ in the local volatility model $(1)$ and denote its price $C_{A}\left(S_{0}, K, T\right)$. We have $\tilde{C}_{A}^{(B S)}\left(S_{0}, K, 0\right)=0$ and $\tilde{C}_{A}^{(B S)}\left(S_{0}, K, \sigma^{2} T\right)>0$ for any $\sigma^{2} T>0$. We have $C_{A}\left(S_{0}, K, T\right)=\tilde{C}_{A}^{(B S)}\left(S_{0}, K, \sigma_{\text {implied }}^{2} T\right) \rightarrow$ 0 as $T \rightarrow 0$. Therefore, $\lim _{T \rightarrow 0} \sigma_{\text {implied }}^{2}\left(S_{0}, K, T\right) T=0$. Hence, by Theorem 2 and Proposition 12 ,

$$
\begin{aligned}
& \lim _{T \rightarrow 0}\left(\sigma_{\text {implied }}^{2}\left(S_{0}, K, T\right) T\right) \log \tilde{C}_{A}^{(B S)}\left(S_{0}, K, \sigma_{\text {implied }}^{2}\left(S_{0}, K, T\right) T\right)=-\mathcal{J}_{B S}\left(K / S_{0}\right), \\
& \lim _{T \rightarrow 0} T \log C_{A}\left(S_{0}, K, T\right)=-\mathcal{I}\left(K, S_{0}\right) .
\end{aligned}
$$

This implies that

$$
\begin{aligned}
\lim _{T \rightarrow 0} \sigma_{\text {implied }}^{2}\left(S_{0}, K, T\right) & =\lim _{T \rightarrow 0} \frac{\sigma_{\text {implied }}^{2}\left(S_{0}, K, T\right) T \log \tilde{C}_{A}^{(B S)}\left(S_{0}, K, \sigma_{\text {implied }}^{2}\left(S_{0}, K, T\right) T\right)}{T \log C_{A}\left(S_{0}, K, T\right)} \\
& =\frac{\mathcal{J}_{B S}\left(K / S_{0}\right)}{\mathcal{I}\left(K, S_{0}\right)} .
\end{aligned}
$$

The same result is obtained for an out-of-the-money Asian put option $K<S_{0}$. The argument follows through in a completely analogous way.

(ii) Next, let us consider the at-the-money Asian call option. ¿From Theorem 6, we have $\lim _{T \rightarrow 0} \frac{1}{\sqrt{T}} C_{A}\left(K, S_{0}, T\right)=\frac{1}{\sqrt{6 \pi}} \sigma\left(S_{0}\right) S_{0}$. Notice that $\tilde{C}_{A}^{(B S)}\left(K, S_{0}, \sigma_{\text {implied }}^{2}\left(K, S_{0}, T\right) T\right)=$ $C_{A}\left(K, S_{0}, T\right)$ and $C_{A}^{(B S)}\left(K, S_{0}, \sigma, T\right)=\tilde{C}_{A}^{(B S)}\left(K, S_{0}, \sigma^{2} T\right)$ can be viewed as a function of $\sigma^{2} T$. Thus, $\lim _{T \rightarrow 0} \frac{\tilde{C}_{A}^{(B S)}\left(K, S_{0}, \sigma_{\text {implied }}^{2}\left(K, S_{0}, T\right) T\right)}{\sqrt{6 \pi} \sqrt{T} \sigma_{\text {implied }}\left(K, S_{0}, T\right)}=S_{0}$. Therefore, $\lim _{T \rightarrow 0} \sigma_{\text {implied }}\left(K, S_{0}, T\right)=$ $\sigma\left(S_{0}\right)$. The same result is obtained for an at-the-money Asian put option.

Proof of Proposition 18. (i) Notice that when $r=q=0, A(T)=S_{0}$ and thanks to the Brownian scaling property, the price of an European option in the Black-Scholes model $\tilde{C}_{E}^{(B S)}\left(K, S_{0}, \sigma^{2} T\right):=\mathbb{E}\left[\left(S_{0} e^{\sigma W_{T}-\frac{1}{2} \sigma^{2} T}-K\right)^{+}\right]$can be viewed as a function of $\sigma^{2} T$. This is a strictly increasing function of this argument. We have by definition of the equivalent log-normal volatility $C_{A}\left(S_{0}, K, T\right)=\tilde{C}_{E}^{(B S)}\left(S_{0}, K, \Sigma_{\mathrm{LN}}^{2} T\right)$ where $C_{A}\left(S_{0}, K, T\right)$ denotes the price of an Asian call option in the local volatility model (1).

We proceed analogously to the proof of Proposition 17. Consider first an out-of-themoney Asian call option $K>S_{0}$, for which we have $C_{A}\left(S_{0}, K, T\right)=\tilde{C}_{E}^{(B S)}\left(S_{0}, K, \Sigma_{\mathrm{LN}}^{2} T\right) \rightarrow$ 0 as $T \rightarrow 0$. Therefore, $\lim _{T \rightarrow 0} \Sigma_{\mathrm{LN}}^{2}\left(S_{0}, K, T\right) T=0$. Hence,

$$
\begin{aligned}
& \lim _{T \rightarrow 0}\left(\Sigma_{\mathrm{LN}}^{2}\left(S_{0}, K, T\right) T\right) \log \tilde{C}_{E}^{(B S)}\left(S_{0}, K, \Sigma_{\mathrm{LN}}^{2}\left(S_{0}, K, T\right) T\right)=-\frac{1}{2} \log ^{2}\left(K / S_{0}\right), \\
& \lim _{T \rightarrow 0} T \log C_{A}\left(S_{0}, K, T\right)=-\mathcal{I}\left(K, S_{0}\right) .
\end{aligned}
$$


This implies that

$$
\begin{aligned}
\lim _{T \rightarrow 0} \Sigma_{\mathrm{LN}}^{2}\left(S_{0}, K, T\right) & =\lim _{T \rightarrow 0} \frac{\Sigma_{\mathrm{LN}}^{2}\left(S_{0}, K, T\right) T \log \tilde{C}_{E}^{(B S)}\left(S_{0}, K, \Sigma_{\mathrm{LN}}^{2}\left(S_{0}, K, T\right) T\right)}{T \log C_{A}\left(S_{0}, K, T\right)} \\
& =\frac{\log ^{2}\left(K / S_{0}\right)}{2 \mathcal{I}\left(K, S_{0}\right)} .
\end{aligned}
$$

The same result is obtained for an out-of-the-money Asian put option $K<S_{0}$.

The corresponding result for the equivalent normal volatility of the Asian option follows from the short maturity relation between the Black-Scholes and Bachelier implied volatilities, see for example Corollary 2 in 34 .

(ii) We use again the fact that the price of an European option in the Black-Scholes model depends only on $\sigma \sqrt{T}$ and denote it as before $\tilde{C}_{E}^{(B S)}\left(K, S_{0}, \sigma^{2} T\right)$. For at-the-money case $S_{0}=K$, we have $\lim _{T \rightarrow 0} \frac{\tilde{C}_{E}^{(B S)}\left(K, S_{0}, \sigma^{2} T\right)}{\sigma \sqrt{2 \pi} \sqrt{T}}=S_{0}$. Therefore, we have

$$
\lim _{T \rightarrow 0} \Sigma_{\mathrm{LN}}\left(K, S_{0}, T\right)=\lim _{T \rightarrow 0} \frac{\frac{C_{A}\left(K, S_{0}, T\right)}{\sqrt{T}}}{\frac{\tilde{C}_{E}^{(B S)}\left(K, S_{0}, \Sigma_{\mathrm{LN}}^{2}\left(K, S_{0}, T\right) T\right)}{\Sigma_{\mathrm{LN}}\left(K, S_{0}, T\right) \sqrt{T}}}=\frac{\frac{1}{\sqrt{6 \pi}} \sigma\left(S_{0}\right) S_{0}}{\frac{1}{\sqrt{2 \pi}} S_{0}}=\frac{1}{\sqrt{3}} \sigma\left(S_{0}\right) .
$$

For the Bachelier model with $K=S_{0}, \mathbb{E}\left[\left(S_{0}+\sigma W_{T}-K\right)^{+}\right]$only depends on $\sigma \sqrt{T}$, and $\lim _{T \rightarrow 0} \frac{1}{\sigma \sqrt{T}} \mathbb{E}\left[\left(S_{0}+\sigma W_{T}-K\right)^{+}\right]=\frac{1}{\sqrt{2 \pi}}$. Therefore,

$$
\lim _{T \rightarrow 0} \Sigma_{\mathrm{N}}\left(K, S_{0}, T\right)=\lim _{T \rightarrow 0} \frac{\frac{C_{A}\left(K, S_{0}, T\right)}{\sqrt{T}}}{\frac{\mathbb{E}\left[\left(S_{0}+\Sigma_{\mathrm{N}}\left(K, S_{0}, T\right) W_{T}-K\right)^{+}\right]}{\Sigma_{\mathrm{N}}\left(K, S_{0}, T\right) \sqrt{T}}}=\frac{\frac{1}{\sqrt{6 \pi}} \sigma\left(S_{0}\right) S_{0}}{\frac{1}{\sqrt{2 \pi}}}=\frac{1}{\sqrt{3}} \sigma\left(S_{0}\right) S_{0} .
$$

We note that these results can also be extracted from Theorem 5.1 of [43].

\subsection{Proofs of the Results in Section 5.}

Proof of Proposition 21. (i) By an argument similar to that used in the proof of Theorem 2 .

$$
\lim _{T \rightarrow 0} T \log C_{f}(T)=\lim _{T \rightarrow 0} T \log \mathbb{P}\left(\kappa S_{T} \geq \int_{0}^{1} S_{t T} d t\right) .
$$

Then, the result follows from the sample path large deviation of $\mathbb{P}\left(S_{t} \cdot \in \cdot\right)$ on $L_{\infty}[0,1]$ and the contraction principle. The asymptotic results for $P(T)$ follows from put-call parity.

(ii) Similar to (i).

(iii) Following the same arguments as in the proof of Theorem 6, we can show that for $\kappa=1$, we have

Claim 1. As $T \rightarrow 0$,

$$
\left|\mathbb{E}\left[\left(e^{(r-q) T} X_{T}-\frac{1}{T} \int_{0}^{T} e^{(r-q) t} X_{t} d t\right)^{+}\right]-\mathbb{E}\left[\left(X_{T}-\frac{1}{T} \int_{0}^{T} X_{t} d t\right)^{+}\right]\right|=O(T) .
$$

Claim 2. As $T \rightarrow 0$,

$$
\mathbb{E}\left[\max _{0 \leq t \leq T}\left|X_{t}-\hat{X}_{t}\right|\right]=O(T)
$$


Claim 3. As $T \rightarrow 0$,

$$
\left|\mathbb{E}\left[\left(X_{T}-\frac{1}{T} \int_{0}^{T} X_{t} d t\right)^{+}\right]-\mathbb{E}\left[\left(\hat{X}_{T}-\frac{1}{T} \int_{0}^{T} \hat{X}_{t} d t\right)^{+}\right]\right|=O(T) .
$$

And Claim 1, Claim 2 and Claim 3 imply that for $\kappa=1$,

$$
\left|C_{f}(T)-\mathbb{E}\left[\left(\kappa \hat{X}_{T}-\frac{1}{T} \int_{0}^{T} \hat{X}_{t} d t\right)^{+}\right]\right|=O(T),
$$

as $T \rightarrow 0$, where we recall that $\hat{X}_{t}=S_{0}+\sigma\left(S_{0}\right) S_{0} W_{t}$. Since $\kappa=1, \kappa \hat{X}_{T}-\frac{1}{T} \int_{0}^{T} \hat{X}_{t} d t$ is a Gaussian random variable with mean zero and variance

$$
\begin{aligned}
& \mathbb{E}\left[\left(\hat{X}_{T}-\frac{1}{T} \int_{0}^{T} \hat{X}_{t} d t\right)^{2}\right] \\
& =\sigma\left(S_{0}\right)^{2} S_{0}^{2} \mathbb{E}\left[\left(W_{T}-\frac{1}{T} \int_{0}^{T} W_{t} d t\right)^{2}\right] \\
& =\sigma\left(S_{0}\right)^{2} S_{0}^{2}\left[\mathbb{E}\left[W_{T}^{2}\right]+\frac{1}{T^{2}} \mathbb{E}\left[\left(\int_{0}^{T} W_{t} d t\right)^{2}\right]-\frac{2}{T} \int_{0}^{T} \mathbb{E}\left[W_{T} W_{t}\right] d t\right] \\
& =\sigma\left(S_{0}\right)^{2} S_{0}^{2}\left[T+\frac{T}{3}-\frac{2}{T} \frac{T^{2}}{2}\right]=\sigma\left(S_{0}\right)^{2} S_{0}^{2} \frac{T}{3} .
\end{aligned}
$$

Hence, we proved the desired result for $C_{f}(T)$. The result for $P_{f}(T)$ is similar.

Proof of Proposition 22. Define a new optimizer function $g(t)=f(t)+\log S_{0}$, satisfying the boundary condition $f(0)=0$ and the constraint $\int_{0}^{1} d t e^{f(t)}=\kappa e^{f(1)}$. Proceeding in a similar fashion as in the proof of Proposition 8, the constraint is taken into account by introducing a Lagrange multiplier $\lambda$ and considering the variational problem associated with the auxiliary functional

$$
\Lambda[f]=\frac{1}{2} \int_{0}^{1}\left(\frac{f^{\prime}(t)}{\sigma\left(S_{0} e^{f(t)}\right)}\right)^{2} d t+\lambda\left(\int_{0}^{1} e^{f(t)} d t-\kappa e^{f(1)}\right) .
$$

By Lemma 28 the solutions of this variational problem satisfy the Euler-Lagrange equation (73) and the transversality condition (75) at $t=1$.

To prove 72 we note that from Lemma 29 we have

$$
\frac{1}{2}\left(\frac{f^{\prime}(t)}{\sigma\left(S_{0} e^{f(t)}\right)}\right)^{2}-\lambda e^{f(t)}=\frac{1}{2} \lambda^{2} \kappa^{2} e^{2 f(1)} \sigma^{2}\left(S_{0} e^{f(1)}\right)-\lambda e^{f(1)} .
$$

We substituted on the right hand side the transversality condition (75). Integrating this relation over $t:(0,1)$ gives the result $(72)$.

Finally, we can eliminate $\lambda$ with the help of the relation (74). This is obtained by comparing two alternative expressions for $f^{\prime}(0)$. First, by integrating the Euler-Lagrange equation (73) over $t:(0,1)$ gives

$$
\frac{f^{\prime}(0)}{\sigma\left(S_{0}\right)}=\lambda\left\{\kappa e^{f(1)} \sigma\left(S_{0} e^{f(1)}\right)-I_{s}[f]\right\}
$$

An alternative relation is obtained by taking $t=0$ in (221). Eliminating $f^{\prime}(0)$ among these two equations gives (74). 
Proof of Proposition 23. The rate function for short-maturity asymptotics of floating strike Asian options in the Black-Scholes model is given by the variational problem

$$
\mathcal{I}_{f}(\kappa)=\inf _{f} \frac{1}{2 \sigma^{2}} \int_{0}^{1}\left[f^{\prime}(t)\right]^{2} d t
$$

where $f(0)=0, f \in \mathcal{A C}[0,1]$ and subject to the constraint

$$
\int_{0}^{1} e^{f(t)} d t=\kappa e^{f(1)}
$$

This can be solved by introducing a Lagrange multiplier $\lambda$ and considering the auxiliary variational problem

$$
\Lambda[f]=\frac{1}{2 \sigma^{2}} \int_{0}^{1}\left[f^{\prime}(t)\right]^{2} d t+\lambda\left(\int_{0}^{1} e^{f(t)} d t-\kappa e^{f(1)}\right),
$$

over all functions satisfying $f(0)=0$.

The solution of the variational problem (225) satisfies the Euler-Lagrange equation

$$
f^{\prime \prime}(t)=\lambda \sigma^{2} e^{f(t)}
$$

which must be solved with the boundary conditions

$$
\begin{array}{ll}
(\mathrm{BC} 1): & f(0)=0, \\
(\mathrm{BC} 2): & f^{\prime}(1)=\lambda \sigma^{2} \kappa e^{f(1)}, \\
(\mathrm{BC} 3): & f^{\prime}(0)=0 .
\end{array}
$$

The boundary condition (BC2) is a transversality condition. The condition (BC3) is new, and follows from the relation of the variational problem for $\Lambda[f]$ to an equivalent variational problem which is obtained by defining a new function $h$ as

$$
f(t)=h(1-t)+f(1) .
$$

Expressed in terms of $h$, the rate function $\mathcal{I}_{f}(\kappa)$ is given by

$$
\mathcal{I}_{f}(\kappa)=\inf _{h} \frac{1}{2 \sigma^{2}} \int_{0}^{1}\left[h^{\prime}(t)\right]^{2} d t
$$

where $h(0)=0, h \in \mathcal{A C}[0,1]$ and it is subject to the constraint

$$
\int_{0}^{1} e^{h(t)} d t=\kappa
$$

This is identical to the variational problem for the rate function $\mathcal{I}\left(\kappa S_{0}, S_{0}\right)$ for fixed strike Asian options (122), in the limit of constant volatility $\sigma(S)=\sigma$. The solution of this variational problem is presented in Proposition 12, and is expressed in terms of the BlackScholes rate function $\mathcal{J}_{\mathrm{BS}}(\kappa)$ as shown in 76 .

For completeness, we give a complete solution of the variational problem (228), and along the way prove the additional boundary condition (BC3). Introducing again a Lagrange multiplier $\eta$, the variational problem (228) can be transformed into an unconstrained optimization of the functional

$$
\Xi[h]=\frac{1}{2 \sigma^{2}} \int_{0}^{1}\left[h^{\prime}(t)\right]^{2} d t+\eta\left(\int_{0}^{1} e^{h(t)} d t-\kappa\right),
$$


over all functions satisfying $h(0)=0$. The solution of this variational problem satisfies the Euler-Lagrange equation

$$
h^{\prime \prime}(t)=\eta \sigma^{2} e^{h(t)}
$$

with the boundary conditions

$$
h(0)=0, \quad h^{\prime}(1)=0 .
$$

The second boundary condition is a transversality condition.

However, $f$ and $h$ are related, so the Euler-Lagrange equations and boundary conditions for $h$ must imply also equivalent conditions on $f$. Comparing the Euler-Lagrange equations for $f$ and $h$ gives

$$
\eta=\lambda e^{f(1)}=\lambda e^{-h(1)} .
$$

Using $f^{\prime}(t)=-h^{\prime}(1-t)$, the transversality condition $h^{\prime}(1)=0$ gives

$$
f^{\prime}(0)=-h^{\prime}(1)=0 .
$$

This proves the boundary condition (BC3) on $f^{\prime}(0)$ quoted above.

Using the additional boundary condition (BC3), it is possible to give a simple expression for the Lagrange multiplier $\lambda$

$$
\lambda=\frac{2}{\sigma^{2} \kappa^{2}} \frac{e^{f(1)}-1}{e^{2 f(1)}} .
$$

This follows from the conservation of the quantity

$$
\frac{1}{2 \sigma^{2}}\left[f^{\prime}(t)\right]^{2}-\lambda \sigma^{2} e^{f(t)}
$$

This gives

$$
-\lambda \sigma^{2}=\frac{1}{2} \lambda^{2} \sigma^{2} \kappa^{2} e^{2 f(1)}-\lambda \sigma^{2} e^{f(1)}
$$

where we used $f^{\prime}(0)$ and the boundary condition $(\mathrm{BC} 2)$ at $t=1$. This concludes the proof of the relation (235).

The solution for $h(1)$ can be found explicitly as shown in the proof of Proposition 12 This is given in equation (198), from which we find

$$
e^{h(1)}= \begin{cases}\cosh ^{2}\left(\frac{1}{2} \beta\right) & \kappa>1, \\ \cos ^{2} \lambda & \kappa<1,\end{cases}
$$

with $\beta, \lambda$ the solutions of the equations $(32)$ and $(33)$, respectively (with the substitutions $\left.K / S_{0} \mapsto \kappa\right)$.

\section{ACKNowledgements}

The authors are grateful to the Editor, an Associate Editor and two anonymous referees for their helpful suggestions that greatly improved the quality of the paper. The authors would like to thank Tai-Ho Wang for helpful discussions, and for bringing to our attention work in progress on a related topic [51]. Lingjiong Zhu is partially supported by NSF Grant DMS-1613164. 


\section{REFERENCES}

[1] Alòs, E., Léon, J. and J. Vives. (2007). On the short-time behavior of the implied volatility for jump-diffusion models with stochastic volatility. Finance and Stochastics. 11, 571-589.

[2] Alziary, B., Decamps, J. P. and P. F. Koehl, A PDE approach to Asian options: Analytical and Numerical evidence. Journal of Banking and Finance. 21, 613-640 (1997).

[3] Andersen, L. and A. Lipton. (2013). Asymptotics for exponential Lévy processes and their volatility smile: Survey and new results. International Journal of Theoretical and Applied Finance. 16, 1350001.

[4] Berestycki, H., Busca, J. and I. Florent. (2002). Asymptotics and calibration of local volatility models. Quantitative Finance. 2, 61-69.

[5] Berestycki, H., Busca, J. and I. Florent. (2004). Computing the implied volatility in stochastic volatility models. Commun. Pure Appl. Math. 57, 1352-1373.

[6] Carr, P., Ewald, C. O. and Y. Xiao. (2008). On the qualitative effect of volatility and duration on prices of Asian options. Finance Research Letters. 5, 162-171.

[7] Carr, P. and M. Schröder. (2003). Bessel processes, the integral of geometric Brownian motion, and Asian options. Theory of Probability and its Applications. 48, 400-425.

[8] Cheng, W., Costanzino, N., Liechty, J., Mazzucato, A. and V. Nistor. (2011). Closed-form asymptotics and numerical approximations of $1 \mathrm{D}$ parabolic equations with applications to option pricing. SIAM J. Fin. Math. 2, 901-934.

[9] Chung, S.L., Shackleton, M. and R. Wojakowski, Efficient quadratic approximation of floating strike Asian option values. Working paper, Lancaster University Management School, 2000

[10] Courant, R. and D. Hilbert. (1953) Methods of Mathematical Physics, vol.1. Interscience Publishers, New York.

[11] Dassios, A. and J. Nagaradjasarma. (2006) The square-root process and Asian options. Quant. Finance 6, 337-347.

[12] Dembo, A. and O. Zeitouni. Large Deviations Techniques and Applications. 2nd Edition, Springer, New York, 1998.

[13] Donati-Martin, C., Ghomrasni, R. and M. Yor. (2001). On certain Markov processes attached to exponential functionals of Brownian motion; application to Asian options. Rev. Math. Iberoam. 17, 179-193.

[14] Dufresne, D. (2000). Laguerre series for Asian and other options. Math. Finance 10, 407-428.

[15] Dufresne, D. (2004). The lognormal approximation in financial and other computations. Adv. Appl. Prob. 36, 747-773.

[16] Dufresne, D. Bessel processes and a functional of Brownian motion, in M. Michele and H. Ben-Ameur (Ed.), Numerical Methods in Finance, 35-57, Springer, 2005.

[17] Dufresne, D. (1990). The distribution of a perpetuity with applications to risk theory and pension funding. Scand. Act. J. 9, 39-79.

[18] Durrleman, V. (2008). Convergence of at-the-money implied volatilities to the spot volatility. J. Appl. Probab. 45, 542-550.

[19] Durrleman, V. (2010). ¿From implied to spot volatilities. Finance and Stochastics. 14, 157-177.

[20] Feng, J., Forde, M. and J.-P. Fouque. (2010). Short maturity asymptotics for a fast mean-reverting Heston stochastic volatility model. SIAM Journal on Financial Mathematics. 1, 126-141.

[21] Feng, J., Fouque, J.-P. and R. Kumar. (2012). Small-time asymptotics for fast mean-reverting stochastic volatility models. Annals of Applied Probability. 22, 1541-1575.

[22] Figueroa-López, J. E. and M. Forde. (2012). The small-maturity smile for exponential Lévy models. SIAM J. Finan. Math. 3, 33-65.

[23] Forde, M. and A. Jacquier. (2009). Small time asymptotics for implied volatility under the Heston model. International Journal of Theoretical and Applied Finance. 12, 861.

[24] Forde, M. and A. Jacquier. (2011). Small time asymptotics for an uncorrelated Local-Stochastic volatility model. Applied Mathematical Finance. 18, 517-535.

[25] Forde, M., Jacquier, A. and R. Lee. (2012). The small-time smile and term structure of implied volatility under the Heston model. SIAM J. Finan. Math. 3, 690-708.

[26] Foschi, P., Pagliarani, S. and A. Pascucci. (2013). Approximations for Asian options in local volatility models. Journal of Computational and Applied Mathematics. 237, 442-459. 
[27] Fu, M., Madan, D. and T. Wang. (1998). Pricing continuous time Asian options: a comparison of Monte Carlo and Laplace transform inversion methods. J. Comput. Finance 2, 49-74.

[28] Gao, K. and R. Lee. (2014). Asymptotics of implied volatility to arbitrary order. Finance and Stochastics. 18, 349-392.

[29] Gatheral, J., Hsu, E. P., Laurent, P., Ouyang, C. and T.-H. Wang. (2012). Asymptotics of implied volatility in local volatility models. Mathematical Finance. 22, 591-620.

[30] Gatheral, J. and T.-H. Wang. (2012). The heat-kernel most-likely-path approximation. IJTAF. 15, 1250001.

[31] Geman, H. and M. Yor. (1993). Bessel processes, Asian options and perpetuities. Math. Finance 3, 349-375.

[32] Gobet, E. and M. Miri. (2014). Weak approximation of averaged diffusion processes. Stoch. Proc. Appl. 124, 475-504.

[33] Guasoni, P. and S. Robertson. (2008). Optimal importance sampling with explicit formulas in continuous time. Fin. Stoch. 12, 1-19.

[34] Grunspan, C. (2011). A note on the equivalence between the normal and lognormal implied volatility: A model free approach. arXiv:1112.1782 math.q-fin.PR]

[35] Henderson, V. and R. Wojakowski. (2002). On the equivalence of floating and fixed-strike Asian options, J. Appl. Prob. 39, 391-394.

[36] Henry-Labordère, P. (2005). A general asymptotic implied volatility for stochastic volatility models. SSRN: 698601.

[37] Ingersoll, J. E. (1987). Theory of Financial Decision Making. Blackwell, Oxford.

[38] Levy, E. (1992). Pricing European average rate currency options. Journal of International Money and Finance. 11, 474-491.

[39] Linetsky, V. (2004). Spectral expansions for Asian (Average price) options. Operations Research 52, 856-867.

[40] Muhle-Karbe, J. and M. Nutz. (2011). Small-time asymptotics of option prices and first absolute moments. J. Appl. Prob. 48, 1003-1020.

[41] Ritchken, P., Sankarasubramanian, L, and A. M. Vijh. (1993). The valuation of path-dependent contracts on the average. Management Science. 39, 1202-1213.

[42] Rogers, L. and Z. Shi. (1995). The value of an Asian option. J. Appl. Prob. 32, 1077-1088.

[43] Roper, M. and M. Rutkowski. (2009). On the relationship between the call price surface and the implied volatility surface close to expiry. IJTAF 12, 427-441.

[44] Shaw, W. T. (2002). Pricing Asian options by contour integration, including asymptotic methods for low volatility. Working paper, University College, London

[45] Shiraya, K., A. Takahashi and M. Toda. (2011). Pricing barrier and average options under stochastic volatility environment. J. Comput. Finance 15, 111-148.

[46] Tankov, P. Pricing and hedging in exponential Lévy models: Review of recent results. In ParisPrinceton Lectures on Mathematical Finance 2010, volume 2003 of Lecture Notes in Math. pages 319-359, Springer, Berlin, 2010.

[47] Varadhan, S. R. S. (1967). Diffusion processes in a small time interval. Communications on Pure and Applied Mathematics. 20, 659-685.

[48] Varadhan, S. R. S. Large Deviations and Applications, SIAM, Philadelphia, 1984.

[49] Vecer, J. (2001). A new PDE approach for pricing arithmetic average Asian options. J. Comput. Finance. 4, 105-113.

[50] Vecer, J. and M. Xu. (2002). Unified Asian pricing. Risk. 15, 113-116.

[51] Wang, T. H., private communication.

E-mail address: dpirjol@gmail.com

Department of Mathematics

Florida State University

1017 ACADEMIC WAY

TAllahassee, FL-32306

United States of AmericA

E-mail address: zhu@math.fsu.edu 